\title{
Bergman 空间上的区域积分算子*
}

\author{
吴志坚 ${ }^{* *}$
}

(Department of Mathematics, The University of Alabama, Tuscaloosa, AL 35487, USA)

摘要刻画单位圆盘 $\mathbb{D}$ 上的非负测度 $\mu$, 它使得从 Bergman 空间 $\mathcal{A}_{p}^{\alpha}$ 到空间 $L^{q}(\partial \mathbb{D})$ 的区域积分算子 $A_{\mu}$ 有界.

\section{关键词 区域积分算子 Bergman 空间 Carleson 测度}

\section{1 引言}

将复平面上的单位圆盘记为 $\mathbb{D}$, 且记

$$
d A(z)=\frac{1}{\pi} d x d y
$$

为 $\mathbb{D}$ 上的单位化 Lebesgue 测度. 令

$$
\alpha>-1, \quad 0<p<\infty .
$$

记

$$
d A_{\alpha}(z)=(\alpha+1)\left(1-|z|^{2}\right)^{\alpha} d A(z) .
$$

Bergman 空间 $\mathcal{A}_{p}^{\alpha}$ 由所有满足下列条件的 $\mathbb{D}$ 上的解析函数 $f$ 组成:

$$
\|f\|_{p, \alpha}^{p}=\|f\|_{\mathcal{A}_{p}^{\alpha}}^{p}=\int_{\mathbb{D}}|f(z)|^{p} d A_{\alpha}(z)<\infty .
$$

单位圆盘 $\mathbb{D}$ 上的所有有界解析函数也记为 $\mathcal{A}_{\infty}^{\alpha}$ 或 $\mathcal{H}^{\infty}$.

将单位圆盘 $\mathbb{D}$ 的边界 (即圆周) 记为 $\partial \mathbb{D}$. 对于任意给定的点 $\zeta \in \partial \mathbb{D}$, 定义单 位圆盘 $\mathbb{D}$ 内以 $\zeta$ 点为顶点的雉 $\Gamma(\zeta)$ 为

$$
\Gamma(\zeta)=\{z \in \mathbb{D}:|\zeta-z|<2(1-|z|)\} .
$$

收稿日期: 2006-01-12; 接受日期: 2006-01-12

本文由葛力明编委推荐

*美国国家科学基金资助项目 (DMS 0200587)

** E-mail: zwu@bama.ua.edu 
令 $\mu$ 为 $\mathbb{D}$ 上的非负测度, Bergman 空间 $\mathcal{A}_{p}^{\alpha}$ 上的区域积分算子是一个由 $\mu$ 导 出的次线性算子, 定义为

$$
A_{\mu}(f)(\zeta)=\int_{\Gamma(\zeta)}|f(z)| \frac{d \mu(z)}{1-|z|}, \quad \forall \zeta \in \partial \mathbb{D} .
$$

区域积分算子在调和分析理论中极为有用，例如，它与非切向极大函数、 Poisson 积分、Littlewood-Paley 算子、乘子、以及帐篷 (tent) 空间等等有密切关 系. Cohn 对 Hardy 空间上的类似的区域积分算子做了系统的研究 ${ }^{[1]}$, 主要结果 是: 对非负测度 $\mu \geqslant 0$ 及参数 $0<p<\infty$, 次线性算子 $A_{\mu}: \mathcal{H}^{p} \rightarrow L^{p}(\partial \mathbb{D})$ 有界的 充要条件为 $\mu$ 是一个 Carleson 测度.

本文的目的是刻画单位圆盘 $\mathbb{D}$ 上的非负测度 $\mu$, 它使得相关的从空间 $\mathcal{A}_{p}^{\alpha}$ 到 空间 $L^{q}=L^{q}(\partial \mathbb{D})$ 的区域积分算子 $A_{\mu}$ 有界, 这里 $0<p, q \leqslant \infty$. 本文的主要结 果是第 3 节中的定理 1 4, 这些结果丰富了对 Bergman 空间上的区域积分算子的 理解.

当 $q=1$ 时, 我们将看到映射 $A_{\mu}: \mathcal{A}_{p}^{\alpha} \rightarrow L^{q}$ 的有界性等价于不等式

$$
\int_{\mathbb{D}}|f(z)| d \mu(z) \leqslant C\|f\|_{p, \alpha}
$$

在 $\mathcal{A}_{p}^{\alpha}$ 上成立. 事实上, 对于某些解析函数空间 $\mathcal{X}$ 及某些参数 $q$, 人们已成功地 刻画了满足如下不等式的非负测度 $\mu$ :

$$
\int_{\mathbb{D}}|f(z)|^{q} d \mu(z) \leqslant C\|f\|_{\mathcal{X}}^{q}, \quad \forall f \in \mathcal{X} .
$$

例如, 读者可参考文献 [2 4](如果 $\mathcal{X}$ 是 Hardy 空间), 文献 [5](如果 $\mathcal{X}$ 是 Bergman 空间), 文献 [6](如果 $\mathcal{X}$ 是 Dirichlet 空间), 这些文章中所引用的相关文章也非常值 得参考.

本文的第 2 节汇集了所需的预备知识和若干技术性结果. 主要定理的叙述 和证明均在第 3 节中. 本文中, $C$ 和 $c$ 代表正常数, 在不同的地方可取不同的 值. 对两个正函数 $a$ 和 $b$, 如果存在正常数 $c$ 和 $C$, 使得 $c a \leqslant b \leqslant C a$, 则称 $a$ 和 $b$ 是等价的, 并记为 $a \asymp b$. 对于正参数 $p \geqslant 1$, 记 $p^{\prime}$ 为 $p$ 的共轭, 即满足方程

$$
\frac{1}{p}+\frac{1}{p^{\prime}}=1 \text {. }
$$

\section{2 预备知识及若干引理}

对于固定的点 $a \in \mathbb{D}$, 令 $\varphi_{a}$ 为单位圆盘 $\mathbb{D}$ 上的 Möbius 变换, 它的特征是将 点 0 和点 $a$ 交换, 例如:

$$
\varphi_{a}(z)=\frac{a-z}{1-\bar{a} z} .
$$

单位圆盘 $\mathbb{D}$ 上的 Bergman 距离定义为

$$
d(z, w)=\frac{1}{2} \log \frac{1+\left|\varphi_{w}(z)\right|}{1-\left|\varphi_{w}(z)\right|}, \quad z, w \in \mathbb{D} .
$$


Bergman 距离是 Möbius 不变的.

对于固定的 $t>0$ 和 $a \in \mathbb{D}$, 定义以 $t$ 为 “半径”, $a$ 为 “圆心” 的 Bergman 圆盘 为

$$
D(a, t)=\{w \in \mathbb{D}: d(w, a)<t\} .
$$

Bergman 圆盘 $D(a, t)$ 事实上是一个 Euclid 圆盘 (通常意义下的圆盘), 且它的圆心 及半径分别是

$$
\tilde{a}=\frac{1-r^{2}}{1-|a|^{2} r^{2}} a, \quad R=r \frac{1-|a|^{2}}{1-|a|^{2} r^{2}}
$$

这里

$$
r=\frac{e^{2 t}-1}{e^{2 t}+1}
$$

将 $D(a, t)$ 在测度 $d A_{\alpha}(z)$ 之下的 “面积” 记为 $|D(a, t)|_{\alpha}$, 则有如下估计:

$$
|D(a, t)|_{\alpha}=\int_{D(a, t)} d A_{\alpha}(z) \asymp(1-|a|)^{2+\alpha} .
$$

对于任意的 $a \in \mathbb{D}$ 和任意的 $z \in D(a, t)$, 如下事实是已知的:

$$
|1-\bar{a} z| \asymp(1-|z|) \asymp(1-|a|) .
$$

与 Bergman 度量相关的分析工具及几何性质已被很好地研究过, 并已在解 析函数空间及算子理论的研究中发挥了有效的作用. 众所周知 ${ }^{[7]}$, 对于任意给定 的 $\delta>0$, 在单位圆盘 $\mathbb{D}$ 中存在着点列 $\left\{z_{j}\right\}$, 称为 $\delta$-网格, 它使得当 $j \neq k$ 时, $d\left(z_{j}, z_{k}\right)>\delta / 5$, 且

$$
\cup_{j} D\left(z_{j}, \delta\right)=\mathbb{D}, \quad \sum_{j} \chi_{D\left(z_{j}, 5 \delta\right)}(z) \leqslant L \quad(\forall z \in \mathbb{D})
$$

成立, 这里 $L>0$ 是一个一致的常数, $\chi_{E}(z)$ 是集合 $E$ 的特征函数.

下述定理是由 Rochberg 给出的:

定理 $\mathbf{A}$ (分解定理) ${ }^{[8]}$ 假设 $\alpha>-1,0<p<\infty$, 则存在一个正数 $\delta_{0}$, 使得对 所有的 $\delta \in\left(0, \delta_{0}\right)$, 单位圆盘 $\mathbb{D}$ 中的任意一个 $\delta$-网格 $\left\{z_{j}\right\}$, 及任意的

$$
m>\max \left\{-1,2\left(1-\frac{1}{p}\right)\right\}
$$

下列各陈述成立:

(A.1) 如果 $f \in \mathcal{A}_{p}^{\alpha}$, 则存在 $\left\{a_{j}\right\} \in l^{p}$ 满足

$$
\left\|\left\{a_{j}\right\}\right\|_{\ell^{p}} \leqslant C\|f\|_{p, \alpha},
$$

使得

$$
f(z)=\sum_{j} a_{j} \frac{\left(1-\left|z_{j}\right|\right)^{m}}{\left(1-\overline{z_{j}} z\right)^{m+\frac{\alpha+2}{p}}} .
$$

(A.2) 如果 $\left\{a_{j}\right\} \in l^{p}$, 则 (A.1) 中级数定义的函数 $f$ 在 $\mathcal{A}_{p}^{\alpha}$ 中收玫, 并且

$$
\|f\|_{p, \alpha} \leqslant C\left\|\left\{a_{j}\right\}\right\|_{\ell^{p}} .
$$


对固定的 $t>0$, 定义 $\mu$ 在 $D(z, t)$ 上的 $\alpha$ - 密度 (平均密度) 为

$$
\rho_{\alpha}(\mu)(z, t)=\frac{\mu(D(z, t))}{|D(z, t)|_{\alpha}}, \quad z \in \mathbb{D} .
$$

密度函数对于研究 Bergman 空间族非常有用. 下述定理是由 Luecking 给出的 (也 可参考文献 [9]):

定理 $\mathbf{B}^{[5]}$ 设 $\alpha>-1,0<q \leqslant p<\infty, \mu$ 是单位圆盘 $\mathbb{D}$ 上的非负测度，则 下列各陈述等价:

(B.1) 存在常数 $C>0$, 使得

$$
\int_{\mathbb{D}}|f|^{q} d \mu \leqslant C\|f\|_{p, \alpha}^{q}, \quad \forall f \in \mathcal{A}_{p}^{\alpha} .
$$

(B.2) 存在 $t>0$, 使得 $\alpha$ - 密度函数

$$
\rho_{\alpha}(\mu)(z, t) \in L^{p /(p-q)}(\mathbb{D}) .
$$

(B.3) 对于任意给定的 $t>0, \alpha$ - 密度函数

$$
\rho_{\alpha}(\mu)(z, t) \in L^{p /(p-q)}(\mathbb{D}) .
$$

应用定理 B, 可以容易地证明下面关于映射 $A_{\mu}: \mathcal{A}_{p}^{\alpha} \rightarrow L^{1}$ 的有界性的刻画:

定理 2.1 令 $\alpha>-1,1 \leqslant p<\infty, \mu$ 是单位圆盘 $\mathbb{D}$ 上的非负测度, 则下列 各陈述等价:

(b.1) 存在常数 $C>0$, 使得

$$
\left\|A_{\mu}(f)\right\|_{L^{1}} \leqslant C\|f\|_{p, \alpha}, \quad \forall f \in \mathcal{A}_{p}^{\alpha} .
$$

(b.2) 存在 $t>0$, 使得 $\alpha$ - 密度函数

$$
\rho_{\alpha}(\mu)(z, t) \in L^{p /(p-1)}(\mathbb{D}) .
$$

(b.3) 对于任意给定的 $t>0, \alpha$ - 密度函数

$$
\rho_{\alpha}(\mu)(z, t) \in L^{p /(p-1)}(\mathbb{D}) \text {. }
$$

证 由 Fubini 定理, 可得

$$
\begin{aligned}
\left\|A_{\mu}(f)\right\|_{L^{1}} & =\int_{\partial \mathbb{D}} \int_{\Gamma(\zeta)}|f(z)| \frac{d \mu(z)}{1-|z|}|d \zeta| \\
& =\int_{\mathbb{D}} \int_{\partial \mathbb{D}} \chi_{\Gamma(\zeta)}(z)|d \zeta||f(z)| \frac{d \mu(z)}{1-|z|} \\
& \asymp \int_{\mathbb{D}}|f(z)| d \mu(z),
\end{aligned}
$$

其中最后的等价估计是由

$$
\int_{\partial \mathbb{D}} \chi_{\Gamma(\zeta)}(z)|d \zeta| \asymp 1-|z|
$$

得到的 (稍后还会引用这个事实 (参见后面的 (2.4) 式)). 由此可见, 所要证明的结 果事实上就是定理 $\mathrm{B}$ 的推论.

设 $g$ 是一个 $L^{1}$ 中的函数, $g$ 在单位圆盘 $\mathbb{D}$ 中的调和扩张 (记为 $P(g)$ ) 可以 表示为下面的 Poisson 积分:

$$
P(g)(z)=\frac{1}{2 \pi} \int_{\partial \mathbb{D}} \frac{1-|z|^{2}}{|\zeta-z|^{2}} g(\zeta)|d \zeta|, \quad \forall z \in \mathbb{D} .
$$


对单位圆周 $\partial \mathbb{D}$ 上的弧 $I$, 记

$$
|I|=\int_{I}|d \zeta| /(2 \pi)
$$

它是 $I$ 的单位化长度. 在单位圆盘 $\mathbb{D}$ 中, 定义以弧 $I$ 为底的 Carleson 方形为

$$
S(I)=\{z \in \mathbb{D}:|z|>1-|I|, z /|z| \in I\} .
$$

对于给定的 $\lambda>0$, 如果存在 $C>0$, 使得对所有的弧 $I \subset \partial \mathbb{D}$, 有

$$
\mu(S(I)) \leqslant C|I|^{\lambda},
$$

则称单位圆盘 $\mathbb{D}$ 上的非负测度 $\mu$ 为 $\lambda$-Carleson 测度. 1-Carleson 测度就是通常的 Carleson 测度. 当 $\lambda>1$ 时， $\lambda$-Carleson 测度也称作 Duren 测度. Carleson 测度 与某些函数空间之间存在着某种自然的联系. 众所周知, 通常意义下的 Carleson 测度与 BMO 空间之间有着密切的关系 (参见文献 [4], p. 240). 如果 $\lambda<1$, 则 $\lambda$-Carleson 测度与 Morrey 空间及 $Q$ 空间有关，其联系可参考文献 [10,11].

如下定理是由 Carleson 给出的:

定理 $\mathbf{C}^{[3]}$ 令 $1<p<\infty, \mu$ 是单位圆盘 $\mathbb{D}$ 上的非负测度，则不等式

$$
\int_{\mathbb{D}}|P(f)(z)|^{p} d \mu(z) \leqslant C\|f\|_{L^{p}}^{p} \quad\left(\forall f \in L^{p}\right)
$$

成立的充要条件为 $\mu$ 是 Carleson 测度.

Hardy 空间 $\mathcal{H}^{p}$ 是由所有满足下列条件的单位圆盘 $\mathbb{D}$ 上的解析函数 $f$ 组成 的:

$$
\|f\|_{\mathcal{H}^{p}}^{p}=\sup _{0<r<1} \int_{\partial \mathbb{D}}|f(r \zeta)|^{p} \frac{|d \zeta|}{2 \pi}<\infty .
$$

如下定理分别由 Carleson ${ }^{[3]}$ 和 Duren ${ }^{[2]}$ 得到, 前者得到了 $p=q$ 的结果, 后 者得到了 $q>p$ 的结果:

定理 $\mathbf{D}$ 令 $0<p \leqslant q<\infty, \mu$ 是单位圆盘 $\mathbb{D}$ 上的非负测度, 则不等式

$$
\int_{\mathbb{D}}|f(z)|^{q} d \mu(z) \leqslant C\|f\|_{\mathcal{H}^{p}}^{q} \quad\left(\forall f \in \mathcal{H}^{p}\right)
$$

成立的充要条件为 $\mu$ 是 $\frac{q}{p}$-Carleson 测度.

对 $g \in L^{1}$, 函数

$$
Q(g)(z)=\frac{\mathrm{i}}{2 \pi} \int_{\partial \mathbb{D}} \frac{\zeta \bar{z}-\bar{\zeta} z}{|\zeta-z|^{2}} g(\zeta)|d \zeta|
$$

不但在 $\mathbb{D}$ 上调和, 而且还与 $P(g)$ 共轭, 也就是说

$$
\mathcal{H}(g)(z)=P(g)(z)+\mathrm{i} Q(g)(z)
$$

在单位圆盘 $\mathbb{D}$ 上解析. 事实上, 当 $1<p<\infty$ 时, 函数 $g$ 属于 $L^{p}$ 等价于 $\mathcal{H}(g)$ 属 于 $\mathcal{H}^{p}$. 从而可以看出定理 $\mathrm{C}$ 和 $\mathrm{D}$ 的联系.

对 $\sigma>0$ 及 $\zeta \in \partial \mathbb{D}$, 定义 $\sigma$ - 雉 $\Gamma_{\sigma}(\zeta)$ 为

$$
\Gamma_{\sigma}(\zeta)=\{z \in \mathbb{D}:|\zeta-z|<(\sigma+1)(1-|z|)\} .
$$


显然 $\Gamma_{1}(\zeta)=\Gamma(\zeta)$. 同时我们指出下列极为有用的估计:

$\int_{\partial \mathbb{D}} \chi_{\Gamma_{\sigma}(\zeta)}(z) \frac{|d \zeta|}{2 \pi}=\frac{2}{\pi} \arcsin \left(\min \left(1,(1-|z|) \sqrt{\frac{\sigma(\sigma+2)}{2|z|}}\right)\right) \asymp 1-|z|, \quad \forall z \in \mathbb{D}$.

假设 $u(z)$ 是单位圆盘 $\mathbb{D}$ 上的调和函数. 对 $\sigma>0$ 及 $\zeta \in \partial \mathbb{D}$, 定义非切向极 大函数 $N_{\sigma}(u)(\zeta)$ 为

$$
N_{\sigma}(u)(\zeta)=\sup _{z \in \Gamma_{\sigma}(\zeta)}|u(z)| .
$$

众所周知，当 $1<p \leqslant \infty$ 时，由 $N_{\sigma}$ 及 $P$ 复合而成的次线性算子 $N_{\sigma} P$ 是 $L^{p}$ 上的 有界算子。

对于单位圆盘 $\mathbb{D}$ 上的测度 $\mu$, 定义 $\mu$ 在圆周 $\partial \mathbb{D}$ 上的调和收缩为

$$
\tilde{\mu}(\zeta)=\frac{1}{2 \pi} \int_{\mathbb{D}} \frac{1-|z|^{2}}{|\zeta-z|^{2}} d \mu(z) .
$$

下面的定理补足了定理 D 中未涉及到的情形, 它可在文献 $[12,13]$ 中找到. 为便于参考, 我们给出它的证明.

定理 2.2 令 $0<q<p \leqslant \infty, \mu$ 是单位圆盘 $\mathbb{D}$ 上的非负测度, 则下列各陈 述等价:

(a) 存在常数 $C>0$, 使得

(b) $\mu$ 在单位圆周上的调和收缩 $\tilde{\mu}$ 属于 $L^{\frac{p}{p-q}}$.

$$
\int_{\mathbb{D}}|f(z)|^{q} d \mu(z) \leqslant C\|f\|_{\mathcal{H}^{p}}^{q}, \quad \forall f \in \mathcal{H}^{p} .
$$

(c) 对于任意固定的 $\sigma>0$, 函数

$$
\hat{\mu}_{\sigma}(\zeta)=\int_{\Gamma_{\sigma}(\zeta)} \frac{d \mu(z)}{1-|z|} \in L^{\frac{p}{p-q}} .
$$

证 首先考虑 $p=\infty$ 的情形. 容易看出, (a) 成立的充分必要条件是 $\mu$ 为有 限测度, 即 $\mu(\mathbb{D})<\infty$. 另一方面, 函数 $\tilde{\mu}$ 和函数 $\hat{\mu}_{\sigma}$ 在 $L^{1}$ 上的范数分别是

$$
\begin{gathered}
\int_{\partial \mathbb{D}} \tilde{\mu}(\zeta)|d \zeta|=\int_{\mathbb{D}} P(1)(z) d \mu(z)=\mu(\mathbb{D}), \\
\int_{\partial \mathbb{D}} \hat{\mu}_{\sigma}(\zeta)|d \zeta|=\int_{\mathbb{D}} \int_{\partial \mathbb{D}} \chi_{\Gamma_{\sigma}(\zeta)}(z)|d \zeta| \frac{d \mu(z)}{1-|z|} \asymp \int_{D} d \mu(z)=\mu(\mathbb{D}),
\end{gathered}
$$

这里，等价符号 “ ”” 是由估计 (2.4) 式得到的. 由此可得到 $p=\infty$ 的结果.

现假设 $p<\infty$. 由 $\mathcal{H}^{p}$ 空间的 Riesz 因子分解理论 (参见文献 [4], p. 56), 我们 知道, 如果 $f \in \mathcal{H}^{p}$ 且 $f \neq \equiv 0$, 则

$$
f(z)=B(z) F(z),
$$

这里 $F \in \mathcal{H}^{p}$ 是一个在单位圆盘 $\mathbb{D}$ 内没有零点的函数，且

$$
\|F\|_{\mathcal{H}^{p}}=\|f\|_{\mathcal{H}^{p}} ;
$$

而 $B(z)$ 则是 Blaschke 乘积. 由此不难看出 (a) 等价于

$$
\int_{\mathbb{D}}|f(z)| d \mu(z) \leqslant C\|f\|_{\mathcal{H}^{p / q}}, \quad \forall f \in \mathcal{H}^{p / q} .
$$


由于 $p / q>1$,算子

$$
\begin{aligned}
\mathcal{H}: L^{p / q} & \rightarrow \mathcal{H}^{p / q}, \\
h & \mapsto P(h)+\mathrm{i} Q(h)
\end{aligned}
$$

是有界的，因而上面的不等式又等价于

$$
\int_{\mathbb{D}}|P(h)(z)| d \mu(z) \leqslant C\|h\|_{L^{p / q}}, \quad \forall h \in L^{p / q}, h \geqslant 0 .
$$

由 Fubini 定理知, 对于函数 $h \geqslant 0$ 且 $h \in L^{p / q}$, 有

$$
\int_{\mathbb{D}} P(h)(z) d \mu(z)=\int_{\mathbb{D}} \int_{\partial \mathbb{D}} \frac{1-|z|^{2}}{|\zeta-z|^{2}} h(\zeta) \frac{|d \zeta|}{2 \pi} d \mu(z)=\int_{\partial \mathbb{D}} \tilde{\mu}(\zeta) h(\zeta)|d \zeta|,
$$

因而, (a) 和 (b) 的等价性可由 $L^{p / q}$ 和 $\left(L^{p / q}\right)^{*}=L^{\frac{p}{p-q}}$ 的对偶关系得到.

(b) $\Rightarrow$ (c) 可由不等式 $\hat{\mu}_{\sigma} \leqslant C \tilde{\mu}$ 得到, 此不等式是

$$
\frac{\chi_{\Gamma_{\sigma}(\zeta)}(z)}{1-|z|} \leqslant 2(\sigma+1) \frac{1-|z|^{2}}{|\zeta-z|^{2}}
$$

的推论, 而后者又可由 $z \in \Gamma_{\sigma}(\zeta)$ 的定义直接导出.

最后证明 (c) $\Rightarrow$ (a). 我们知道, 当 $p / q>1$ 时, 算子 $N_{\sigma} P$ 在 $L^{p / q}$ 上是有界 的. 对 $h \in L^{p / q}$ 且 $h \geqslant 0$, 由 Fubini 定理与等价估计 (2.4) 式, 可以得到

$$
\begin{aligned}
\int_{\mathbb{D}} P(h)(z) d \mu(z) & \asymp \int_{\partial \mathbb{D}} \int_{\Gamma_{\sigma}(\zeta)} P(h)(z) \frac{d \mu(z)}{1-|z|}|d \zeta| \\
& \leqslant \int_{\partial \mathbb{D}} N_{\sigma} P(h)(\zeta) \hat{\mu}(\zeta)|d \zeta| \\
& \leqslant\left\|N_{\sigma} P(h)\right\|_{L^{p / q}}\left\|\hat{\mu}_{\sigma}\right\|_{L^{(p / q)^{\prime}}} \quad \text { (Hölder 不等式) } \\
& \leqslant C\|h\|_{L^{p / q}}\left\|\hat{\mu}_{\sigma}\right\|_{L^{\frac{p}{p-q}}} .
\end{aligned}
$$

将 $\sigma>0$ 视为雉 $\Gamma_{\sigma}(\zeta)$ 的参数. 可以看出, 雉 $\Gamma_{\sigma}(\zeta)$ 作为一个集合, 随 $\sigma$ 的 递增而增大. 如下结果定量地给出了这种递增的 Bergman 几何意义:

引理 2.3 设 $\sigma>0, t \geqslant 0, \zeta \in \partial \mathbb{D}$. 令

$$
\sigma_{t}=(\sigma+2) e^{2 t}-2,
$$

则

$$
D(z, t) \subset \Gamma_{\sigma_{t}}(\zeta), \quad \forall z \in \Gamma_{\sigma}(\zeta) .
$$

证 令 $w \in D(z, t), z \in \Gamma_{\sigma}(\zeta)$, 只需证明 $w \in \Gamma_{\sigma_{t}}(\zeta)$. 首先注意到

$$
\begin{aligned}
|\zeta-w| & \leqslant|\zeta-z|+|z-w| \\
& <(\sigma+1)(1-|z|)+|z-w| \\
& \leqslant(\sigma+1)(1-|w|+|z-w|)+|z-w| \\
& =(\sigma+1)(1-|w|)+(\sigma+2)|z-w| .
\end{aligned}
$$

记

$$
r=\frac{e^{2 t}-1}{e^{2 t}+1}
$$


Bergman 圆盘 $D(w, t)$ 的 Euclid 圆心及半径分别是

$$
\tilde{w}=\frac{1-r^{2}}{1-|w|^{2} r^{2}} w, \quad R=r \frac{1-|w|^{2}}{1-|w|^{2} r^{2}} .
$$

由于 $w \in D(z, t)$ 与 $z \in D(w, t)$ 等价, 故可得

$$
\begin{aligned}
|z-w| & \leqslant|z-\tilde{w}|+|\tilde{w}-w| \\
& <r \frac{1-|w|^{2}}{1-|w|^{2} r^{2}}+|w|\left(1-\frac{1-r^{2}}{1-|w|^{2} r^{2}}\right) \\
& =\frac{r\left(1-|w|^{2}\right)}{1-|w| r},
\end{aligned}
$$

因此

$$
\begin{aligned}
|\zeta-w| & <(\sigma+1)(1-|w|)+(\sigma+2) \frac{r\left(1-|w|^{2}\right)}{1-|w| r} \\
& <\left(\sigma+1+(\sigma+2) \frac{2 r}{1-r}\right)(1-|w|) \\
& =\left((\sigma+2) e^{2 t}-1\right)(1-|w|) .
\end{aligned}
$$

对 $\sigma>0$ 及固定的 $z \in \mathbb{D}$, 定义圆周 $\partial \mathbb{D}$ 上的弧

$$
I_{\sigma}(z)=\left\{\zeta \in \partial \mathbb{D}: z \in \Gamma_{\sigma}(\zeta)\right\} .
$$

显然, 对 $z \in \mathbb{D} \backslash\{0\}$ 而言, 弧 $I_{\sigma}(z)$ 在圆周 $\partial \mathbb{D}$ 上的中点是 $z /|z|$, 且它的单位化 长度可借助等价式 (2.4) 估计如下:

$$
\left|I_{\sigma}(z)\right|=\int_{I_{\sigma}(z)} \frac{|d \zeta|}{2 \pi}=\int_{\partial \mathbb{D}} \chi_{\Gamma_{\sigma}(\zeta)}(z) \frac{|d \zeta|}{2 \pi} \asymp(1-|z|) .
$$

对 $g \in L^{1}$, 定义 $g$ 在单位圆盘 $\mathbb{D}$ 上的另一种扩张为

$$
T_{\sigma}(g)(z)=\frac{1}{\left|I_{\sigma}(z)\right|} \int_{I_{\sigma}(z)} g(\zeta) \frac{|d \zeta|}{2 \pi}, \quad \forall z \in \mathbb{D} .
$$

如果 $g \geqslant 0$, 函数 $T_{\sigma}(g)$ 可用作 $P(g)$ 即 $g$ 的调和扩张的下估计, 这是下面的定理 所要证明的:

定理 2.4 令 $\sigma>0$, 则存在常数 $C_{\sigma}>0$, 使得对于任意的 $g \geqslant 0$ 且 $g \in L^{1}$, 如下不等式成立:

$$
T_{\sigma}(g)(z) \leqslant C_{\sigma} P(g)(z), \quad \forall z \in \mathbb{D} .
$$

证 我们知道 $g$ 在 $\mathbb{D}$ 上的调和扩张是

$$
P(g)(z)=\frac{1}{2 \pi} \int_{\partial \mathbb{D}} \frac{1-|z|^{2}}{|\zeta-z|^{2}} g(\zeta)|d \zeta|,
$$

因此只需要建立估计

$$
\frac{1}{\left|I_{\sigma}(z)\right|} \chi_{I_{\sigma}(z)}(\zeta) \leqslant C_{\sigma} \frac{1-|z|^{2}}{|\zeta-z|^{2}}, \quad \forall z \in \mathbb{D}, \quad \forall \zeta \in \partial \mathbb{D},
$$

而这是简单的.

与定理 2.4 相反, 如下结果在一定意义上给出了 $P(g)$ 的上估计: 
定理 2.5 令 $\sigma>0, t \geqslant 0$,

$$
\sigma_{t}=(\sigma+2) e^{2 t}-2,
$$

则对任意的 $g \in L^{1}$, 如下不等式成立:

$$
|P(g)(w)| \leqslant T_{\sigma} N_{\sigma_{t}} P(g)(z), \quad \forall w \in D(z, t), \quad \forall z \in \mathbb{D} .
$$

证 很显然,

$$
|P(g)(w)| \leqslant N_{\sigma_{t}} P(g)(\zeta), \quad \forall \zeta \in I_{\sigma_{t}}(w) .
$$

由引理 2.3 可得, 如果 $w \in D(z, t)$, 则 $I_{\sigma}(z) \subset I_{\sigma_{t}}(w)$. 将上面的不等式的两边对 $|d \zeta|$ 在 $I_{\sigma}(z)$ 上积分, 就可以得到所要的结果。

引理 2.6 令 $\alpha>-1,0<p<\infty, t>0$, 则存在常数 $C>0$, 使得对单位圆 盘 $\mathbb{D}$ 上的所有解析函数 $f$ 及任意 $z \in \mathbb{D}$, 如下不等式成立:

$$
|f(z)|^{p} \leqslant \frac{C}{|D(z, t)|_{\alpha}} \int_{D(z, t)}|f(w)|^{p} d A_{\alpha}(w) .
$$

此外, 如果 $1 \leqslant p<\infty$, 则这个不等式对于 $\mathbb{D}$ 上的调和函数 $f$ 也成立.

证 显而易见, $w \in D(0, t)$ 当且仅当 $\varphi_{z}(w) \in D(z, t)$. 由解析 (调和) 函数在 $D(0, t)$ 上的均值公式、积分变量代换 $\eta=\varphi_{z}(w)$ 及等价估计 (2.2) 式就可以证明 所需要的结果了.

对 $\tau>0$ 及单位圆盘 $\mathbb{D}$ 上的非负测度 $\mu$, 考虑更为广泛的区域积分算子

$$
A_{\mu}^{\tau}(f)(\zeta)=\int_{\Gamma_{\tau}(\zeta)}|f(z)| \frac{d \mu(z)}{1-|z|} .
$$

显然 $A_{\mu}^{1}=A_{\mu}$, 而且当 $0<\tau_{1}<\tau_{2}$ 时, 在圆周 $\partial \mathbb{D}$ 上有

$$
A_{\mu}^{\tau_{1}}(f) \leqslant A_{\mu}^{\tau_{2}}(f) \text {. }
$$

引理 2.7 令 $\sigma, t>0$,

$$
\sigma_{t}=(\sigma+2) e^{2 t}-2,
$$

则存在常数 $C>0$, 使得对于单位圆盘 $\mathbb{D}$ 上的任意解析函数 $f$ 及任意 $\zeta \in \partial \mathbb{D}$, 有

$$
\begin{aligned}
A_{\mu}^{\sigma}(f)(\zeta) & =\int_{\Gamma_{\sigma}(\zeta)}|f(z)| \frac{d \mu(z)}{1-|z|} \\
& \leqslant C \int_{\Gamma_{\sigma_{t}}(\zeta)}|f(z)| \frac{\rho_{\alpha}(\mu)(z, t) d A_{\alpha}(z)}{1-|z|} .
\end{aligned}
$$

证 由引理 2.6 和 2.3 及等式

$$
\chi_{D(z, t)}(w)=\chi_{D(w, t)}(z)
$$

可得

$$
\begin{aligned}
& A_{\mu}^{\sigma}(f)(\zeta) \\
\leqslant & \int_{\Gamma_{\sigma}(\zeta)} \frac{C}{|D(z, t)|_{\alpha}} \int_{D(z, t)}|f(w)| d A_{\alpha}(w) \frac{d \mu(z)}{1-|z|} \\
= & \int_{\mathbb{D}} \int_{\Gamma_{\sigma}(\zeta)} \frac{C}{|D(z, t)|_{\alpha}} \chi_{D(z, t)}(w)|f(w)| \frac{d \mu(z)}{1-|z|} d A_{\alpha}(w) \quad \text { (由 Fubini 定理) }
\end{aligned}
$$




$$
\begin{aligned}
& \leqslant \int_{\Gamma_{\sigma_{t}}(\zeta)} \int_{\Gamma_{\sigma}(\zeta)} \frac{C}{|D(z, t)|_{\alpha}} \chi_{D(w, t)}(z)|f(w)| \frac{d \mu(z)}{1-|z|} d A_{\alpha}(w) \\
& \asymp \int_{\Gamma_{\sigma_{t}}(\zeta)}|f(w)| \frac{\rho_{\alpha}(\mu)(w, t) d A_{\alpha}(w)}{1-|w|} \text { (由 (2.2) 式), }
\end{aligned}
$$

由此即得结论.

如果 $\mu$ 是单位圆盘 $\mathbb{D}$ 上的非负测度，则 $\mu$ 所诱导的在小 Bergman 圆盘上 的 $\alpha$ - 密度函数可被大 Bergman 圆盘上的 $\alpha$ - 密度函数所控制. 准确地说, 对测度 $\mu \geqslant 0$ 及固定的 $r_{1}$ 和 $r_{2}: 0<r_{1}<r_{2}$, 存在常数 $C>0$, 使得对于任意满足

$$
D\left(z_{1}, r_{1}\right) \subset D\left(z_{2}, r_{2}\right) \subset \mathbb{D}
$$

的 $\left(z_{1}, r_{1}\right)$ 和 $\left(z_{2}, r_{2}\right)$, 如下估计成立:

$$
\rho_{\alpha}(\mu)\left(z_{1}, r_{1}\right) \leqslant C \rho_{\alpha}(\mu)\left(z_{2}, r_{2}\right) .
$$

事实上, 由等价估计 (2.2) 式可得

$$
\left(1-\left|z_{1}\right|\right) \asymp\left(1-\left|z_{2}\right|\right),
$$

因此估计 (2.6) 可由不等式

$$
\mu\left(D\left(z_{1}, r_{1}\right)\right) \leqslant \mu\left(D\left(z_{2}, r_{2}\right)\right)
$$

得到.

下面的结果说明，测度 $\mu$ 所诱导的在大 Bergman 圆盘上的 $\alpha$ - 密度函数可被 在小 Bergman 圆盘上的 $\alpha$ - 密度函数的积分均值所控制.

引理 2.8 令 $\alpha>-1, r>0,0<s<t$, 则存在常数 $C>0$, 使得对于单位圆 盘 $\mathbb{D}$ 上的任意非负测度 $\mu$ 及任意 $a \in \mathbb{D}$, 如下估计成立:

$$
\begin{aligned}
\frac{1}{C} \rho_{\alpha}(\mu)(a, r+s) & \leqslant \frac{1}{|D(a, r)|_{\alpha}} \int_{D(a, r)} \rho_{\alpha}(\mu)(z, t) d A_{\alpha}(z) \\
& \leqslant C \rho_{\alpha}(\mu)(a, r+t) .
\end{aligned}
$$

证 由于有估计 (2.1) 和 (2.2) 式, 且当 $z \in D(a, r)$ 时, 有

$$
D(z, t) \subset D(a, r+t)
$$

(从而

$$
\mu(D(z, t)) \leqslant \mu(D(a, r+t))),
$$

可得到引理的第 2 个不等式. 对于第 1 个不等式, 只需要证明

$$
\mu(D(a, r+s)) \leqslant \frac{C}{|D(a, r)|_{\alpha}} \int_{D(a, r)} \mu(D(z, t)) d A_{\alpha}(z), \quad \forall a \in \mathbb{D} .
$$

我们知道，作为集合，有

$$
D(a, r+t)=\cup_{z \in D(a, r)} D(z, t) .
$$


由 Fubini 定理, 可以得到

$$
\begin{aligned}
& \int_{D(a, r)} \mu(D(z, t)) d A_{\alpha}(z) \\
= & \int_{D(a, r)} \int_{D(a, r+t)} \chi_{D(z, t)}(w) d \mu(w) d A_{\alpha}(z) \\
= & \int_{D(a, r+t)} \int_{D(a, r)} \chi_{D(w, t)}(z) d A_{\alpha}(z) d \mu(w) \\
\geqslant & \int_{D(a, r+s)} \int_{D(a, r)} \chi_{D(w, t)}(z) d A_{\alpha}(z) d \mu(w) \\
\geqslant & \mu(D(a, r+s)) \inf _{w \in D(a, r+s)}|D(a, r) \cap D(w, t)|_{\alpha} .
\end{aligned}
$$

很显然, 集合 $D(a, r) \cap D(w, t)$ 含有一个半径为 $(t-s) / 2$ 的 Bergman 圆盘 $D$, 因 此由等价估计 (2.2) 式可得

$$
|D(a, r) \cap D(w, t)|_{\alpha} \geqslant|D|_{\alpha} \asymp(1-|a|)^{\alpha+2} .
$$

\section{3 主要结果}

在上一节中, 对 $\sigma>0$ 及单位圆盘 $\mathbb{D}$ 上的非负测度 $\mu$, 定义了

$$
A_{\mu}^{\sigma}(f)(\zeta)=\int_{\Gamma_{\sigma}(\zeta)}|f(z)| \frac{d \mu(z)}{1-|z|}, \quad \forall \zeta \in \partial \mathbb{D} .
$$

本节中的所有定理都是针对这类一般性的区域积分算子的. 定理 1 和 2 对应 $p \leqslant q$ 的情形, 而定理 3 和 4 则对应 $p>q$ 的情形.

对一个单位圆盘 $\mathbb{D}$ 内的 $\delta$-网格 $\left\{z_{j}\right\}$, 记

$$
D_{j}=D\left(z_{j}, \delta\right), \quad \tilde{D}_{j}=D\left(z_{j}, 2 \delta\right) .
$$

定理 1 令 $\alpha>-1,0<p \leqslant q \leqslant \infty, 0<p \leqslant 1$. 对单位圆盘 $\mathbb{D}$ 上的非负测度 $\mu$ 而言, 下列各陈述等价:

(1a) $A_{\mu}$ 从 $\mathcal{A}_{p}^{\alpha}$ 到 $L^{q}$ 有界.

(1b) 存在 $\sigma>0$, 使得 $A_{\mu}^{\sigma}$ 从 $\mathcal{A}_{p}^{\alpha}$ 到 $L^{q}$ 有界.

(1c) 存在 $\delta>0$, 使得 $\alpha$ - 密度函数 $\rho_{\alpha}(\mu)(z, \delta)$ 满足

$$
\rho_{\alpha}(\mu)(z, \delta) \leqslant C(1-|z|)^{(\alpha+2)\left(\frac{1}{p}-1\right)+1-\frac{1}{q}}, \quad \forall z \in \mathbb{D} .
$$

(1d) 对于任意固定的 $t>0, \alpha$ - 密度函数 $\rho_{\alpha}(\mu)(z, t)$ 满足 (1c) 中的不等式.

注 1 单位圆盘 $\mathbb{D}$ 上的解析函数序列 $\left\{g_{j}\right\}$ 称为在 $\mathcal{A}_{p}^{\alpha}$ 中弱收玫到 0 , 如果它 在 $\mathcal{A}_{p}^{\alpha}$ 中有界, 且在 $\mathbb{D}$ 中的任意紧子集上一致收玫到 $0 . A_{\mu}^{\sigma}$ 称为从 $\mathcal{A}_{p}^{\alpha}$ 到 $L^{q}$ 上 的紧算子, 如果对于任意 $\mathcal{A}_{p}^{\alpha}$ 中弱收玫到 0 的解析函数序列 $\left\{g_{j}\right\}$, 都有 $A_{\mu}^{\sigma}\left(g_{j}\right)$ 在 $L^{q}$ 中收敛到 0 .

同定理 1 的假设, 将各陈述中的 “有界” 换为 “紧”, 且将 (1c) 中 $\alpha$ - 密度函数 $\rho_{\alpha}(\mu)(z, \delta)$ 所应满足的条件换为

$$
\frac{\rho_{\alpha}(\mu)(z, \delta)}{(1-|z|)^{(\alpha+2)\left(\frac{1}{p}-1\right)+1-\frac{1}{q}}} \rightarrow 0 \quad(|z| \rightarrow 1),
$$


我们就可以得到关于 $A_{\mu}^{\sigma}$ 是从 $\mathcal{A}_{p}^{\alpha}$ 到 $L^{q}$ 上的紧算子的相关结果了, 其证明和下 面定理 1 的证明类似.

定理 1 的证 只证明 (1b) $\Rightarrow(1 \mathrm{c})$ 和 (1d) $\Rightarrow(1 \mathrm{a})$, 因为 (1c) $\Rightarrow(1 \mathrm{~d})$ 是引理 2.8 和估计 (2.6) 式的推论, 而其余要证的都很简单.

假设 (1b) 成立. 令 $a \in \mathbb{D}, m>0$. 考虑检验函数

$$
f_{a}(z)=\frac{(1-|a|)^{m}}{(1-\bar{a} z)^{m+\frac{\alpha+2}{p}}} .
$$

显而易见

$$
\left\|f_{a}\right\|_{p, \alpha} \asymp 1,
$$

因此

$$
\left\|A_{\mu}^{\sigma}\left(f_{a}\right)\right\|_{L^{q}} \leqslant C .
$$

此外, 对固定的 $\delta>0$, 由估计 (2.2) 式可得

$$
\left|f_{a}(z)\right| \asymp(1-|a|)^{-\frac{\alpha+2}{p}}, \quad \forall z \in D(a, \delta) .
$$

对 (1b) 中的 $\sigma$, 选择 $\delta>0$, 使得

$$
2\left(e^{2 \delta}-1\right)<\sigma,
$$

则

$$
\tau=(\sigma+2) e^{-2 \delta}-2>0 .
$$

由引理 2.3 可知, 当 $a \in \Gamma_{\tau}(\zeta)$ 时, 有

$$
D(a, \delta) \subset \Gamma_{\sigma}(\zeta) .
$$

如果 $q<\infty$, 则有

$$
\left|I_{\tau}(a)\right| \asymp(1-|a|),
$$

由此及估计 (3.1) 式可得

$$
\begin{aligned}
(1-|a|)\left(\frac{\mu(D(a, \delta))}{(1-|a|)^{1+\frac{\alpha+2}{p}}}\right)^{q} & \leqslant C \int_{I_{\tau}(a)}\left(\int_{D(a, \delta)}\left|f_{a}(z)\right| \frac{d \mu(z)}{1-|z|}\right)^{q}|d \zeta| \\
& \leqslant C \int_{\partial \mathbb{D}}\left(\int_{\Gamma_{\sigma}(\zeta)}\left|f_{a}(z)\right| \frac{d \mu(z)}{1-|z|}\right)^{q}|d \zeta| \\
& =C\left\|A_{\mu}^{\sigma}\left(f_{a}\right)\right\|_{L^{q}}^{q} \leqslant C\left\|f_{a}\right\|_{p, \alpha},
\end{aligned}
$$

此不等式等价于

$$
\rho_{\alpha}(\mu)(a, \delta) \leqslant C(1-|a|)^{(\alpha+2)\left(\frac{1}{p}-1\right)+1-\frac{1}{q}} .
$$

如果 $q=\infty$, 则有

$$
\begin{aligned}
\frac{\mu(D(a, \delta))}{(1-|a|)^{1+\frac{\alpha+2}{p}}} & \leqslant C \int_{D(a, \delta)}\left|f_{a}(z)\right| \frac{d \mu(z)}{1-|z|} \leqslant C \int_{\Gamma_{\sigma}(\zeta)}\left|f_{a}(z)\right| \frac{d \mu(z)}{1-|z|} \\
& =C\left\|A_{\mu}^{\sigma}\left(f_{a}\right)\right\|_{L^{\infty}} \leqslant C\left\|f_{a}\right\|_{p, \alpha},
\end{aligned}
$$


此不等式等价于

$$
\rho_{\alpha}(\mu)(a, \delta) \leqslant C(1-|a|)^{(\alpha+2)\left(\frac{1}{p}-1\right)+1},
$$

因此 $(1 b) \Rightarrow(1 c)$ 得证.

假设 $(1 \mathrm{~d})$ 成立. 令 $\left\{z_{j}\right\}$ 为单位圆盘 $\mathbb{D}$ 内的一个 $\delta$-网格, 且 $\delta$ 小于 (1d) 中的 $t$. 由等价估计 (2.2) 式及 (1d) 中关于 $\rho_{\alpha}(\mu)(z, t)$ 的假设, 可得

$$
\begin{aligned}
\frac{\mu\left(D_{j}\right)}{1-\left|z_{j}\right|} & \asymp\left(1-\left|z_{j}\right|\right)^{1+\alpha} \rho_{\alpha}(\mu)\left(z_{j}, \delta\right) \\
& \leqslant C\left(1-\left|z_{j}\right|\right)^{1+\alpha} \rho_{\alpha}(\mu)\left(z_{j}, t\right) \\
& \leqslant C\left(1-\left|z_{j}\right|\right)^{\frac{(\alpha+2)}{p}-\frac{1}{q}} .
\end{aligned}
$$

因而，对于单位圆盘 $\mathbb{D}$ 上的任意解析函数 $f$, 有

$$
\begin{aligned}
A_{\mu}(f)(\zeta) & \leqslant C \sum_{j: D_{j} \cap \Gamma(\zeta) \neq \emptyset} \sup _{z \in D_{j}}|f(z)| \frac{\mu\left(D_{j}\right)}{1-\left|z_{j}\right|} \\
& \leqslant C \sum_{j: D_{j} \cap \Gamma(\zeta) \neq \emptyset}\left(1-\left|z_{j}\right|\right)^{\frac{(\alpha+2)}{p}-\frac{1}{q}} \sup _{z \in D_{j}}|f(z)| .
\end{aligned}
$$

由于 $0<p \leqslant 1$, 由上面的不等式可进一步导出

$$
\left(A_{\mu}(f)(\zeta)\right)^{p} \leqslant C \sum_{j: D_{j} \cap \Gamma(\zeta) \neq \emptyset}\left(1-\left|z_{j}\right|\right)^{\alpha+2-\frac{p}{q}} \sup _{z \in D_{j}}|f(z)|^{p} .
$$

对 $z \in D_{j}$, 显然有 $D(z, \delta) \subset \tilde{D}_{j}$, 进而由估计 (2.1) 式可得

$$
|D(z, \delta)|_{\alpha} \asymp\left|\tilde{D}_{j}\right|_{\alpha},
$$

因此, 根据引理 2.6, 当 $z \in D_{j}$ 时, 就有

$$
\begin{aligned}
|f(z)|^{p} & \leqslant \frac{C}{|D(z, \delta)|_{\alpha}} \int_{D(z, \delta)}|f(w)|^{p} d A_{\alpha}(w) \\
& \leqslant \frac{C}{\left|\tilde{D}_{j}\right|_{\alpha}} \int_{\tilde{D}_{j}}|f(w)|^{p} d A_{\alpha}(w) .
\end{aligned}
$$

再由估计 (2.1) 和 (2.2) 式, 就可得到

$$
\left(1-\left|z_{j}\right|\right)^{\alpha+2-\frac{p}{q}} \sup _{z \in D_{j}}|f(z)|^{p} \leqslant C \int_{\tilde{D}_{j}}(1-|w|)^{-p / q}|f(w)|^{p} d A_{\alpha}(w) .
$$

进一步假设

$$
\sigma=3 e^{6 \delta}-2
$$

如果

$$
D_{j} \cap \Gamma(\zeta) \neq \emptyset, \quad z \in D_{j} \cap \Gamma(\zeta)
$$

则由引理 2.3 可得

$$
\tilde{D}_{j} \subset D(z, 3 \delta) \subset \Gamma_{\sigma}(\zeta),
$$

因此, 由上面的各不等式及估计 (2.3) 式, 得 


$$
\begin{aligned}
\left(A_{\mu}(f)(\zeta)\right)^{p} & \leqslant C \sum_{j: D_{j} \cap \Gamma(\zeta) \neq \emptyset} \int_{\tilde{D}_{j}}(1-|w|)^{-p / q}|f(w)|^{p} d A_{\alpha}(w) \\
& \leqslant C L \int_{\Gamma_{\sigma}(\zeta)}|f(z)|^{p}(1-|z|)^{-p / q} d A_{\alpha}(z) .
\end{aligned}
$$

如果 $q<\infty$, 则应用 Hölder 不等式得

$$
\begin{aligned}
\left(A_{\mu}(f)(\zeta)\right)^{q} & \leqslant C\left(\int_{\Gamma_{\sigma}(\zeta)}|f(z)|^{p}(1-|z|)^{-p / q} d A_{\alpha}(z)\right)^{q / p} \\
& \leqslant C \int_{\Gamma_{\sigma}(\zeta)}|f(z)|^{p} \frac{d A_{\alpha}(z)}{1-|z|}\left(\int_{\Gamma_{\sigma}(\zeta)}|f(z)|^{p} d A_{\alpha}(z)\right)^{(q-p) / p} \\
& \leqslant C\|f\|_{p, \alpha}^{q-p} \int_{\Gamma_{\sigma}(\zeta)}|f(z)|^{p} \frac{d A_{\alpha}(z)}{1-|z|}
\end{aligned}
$$

因而得到

$$
\begin{aligned}
\left\|A_{\mu}(f)\right\|_{L^{q}}^{q} & \leqslant C\|f\|_{p, \alpha}^{q-p} \int_{\partial \mathbb{D}} \int_{\Gamma_{\sigma}(\zeta)}|f(z)|^{p} \frac{d A_{\alpha}(z)}{1-|z|}|d \zeta| \\
& =C\|f\|_{p, \alpha}^{q-p} \int_{\mathbb{D}} \int_{\partial \mathbb{D}} \chi_{\Gamma_{\sigma}(\zeta)}(z)|d \zeta||f(z)|^{p} \frac{d A_{\alpha}(z)}{1-|z|} \quad \text { (由 Fubini 定理) } \\
& \leqslant C\|f\|_{p, \alpha}^{q} .
\end{aligned}
$$

如果 $q=\infty$, 则可直接得到

$$
A_{\mu}(f)(\zeta) \leqslant C\left(\int_{\Gamma_{\sigma}(\zeta)}|f(z)|^{p} d A_{\alpha}(z)\right)^{1 / p} \leqslant C\|f\|_{p, \alpha} .
$$

定理 2 令 $\alpha>-1,1<p \leqslant q<\infty$. 对单位圆盘 $\mathbb{D}$ 上的非负测度 $\mu$ 而言, 下列各陈述等价:

(2a) $A_{\mu}$ 从 $\mathcal{A}_{p}^{\alpha}$ 到 $L^{q}$ 有界.

(2b) 存在 $\sigma>0$, 使得 $A_{\mu}^{\sigma}$ 从 $\mathcal{A}_{p}^{\alpha}$ 到 $L^{q}$ 有界.

(2c) 存在 $\delta>0$, 使得由 $\alpha$ - 密度函数 $\rho_{\alpha}(\mu)(z, \delta)$ 所诱导的测度

$$
\left(\rho_{\alpha}(\mu)(z, \delta)\right)^{\frac{p}{p-1}} d A_{\alpha}(z)
$$

是一个 $\frac{p(q-1)}{q(p-1)}$-Carleson 测度.

(2d) 对任意固定的 $t>0, \alpha$ - 密度函数 $\rho_{\alpha}(\mu)(z, t)$ 具有上述性质.

注 2 单位圆盘 $\mathbb{D}$ 上的非负测度 $\nu$ 称为紧的 $\lambda$-Carleson 测度, 如果它是 $\lambda$ Carleson 测度，并且有

$$
\frac{\nu(S(I))}{|I|^{\lambda}} \rightarrow 0 \quad(|I| \rightarrow 0) .
$$

同定理 2 的假设, 将各陈述中的 “有界” 换为 “紧”, 且将 (2c) 中 $\alpha$ - 密度函数 $\rho_{\alpha}(\mu)(z, \delta)$ 所应满足的条件换为: 测度

$$
\left(\rho_{\alpha}(\mu)(z, \delta)\right)^{\frac{p}{p-1}} d A_{\alpha}(z)
$$

是一个紧的 $\frac{p(q-1)}{q(p-1)}$-Carleson 测度, 就可以得到关于 $A_{\mu}^{\sigma}$ 是从 $\mathcal{A}_{p}^{\alpha}$ 到 $L^{q}$ 上的紧算子 的相关结果，其证明和下面定理 2 的证明类似. 
定理 2 的证 只需证明 $(2 b) \Rightarrow(2 c) \Rightarrow(2 d) \Rightarrow(2 a)$, 其余的均不难.

假设 $A_{\mu}^{\sigma}$ 从 $\mathcal{A}_{p}^{\alpha}$ 到 $L^{q}$ 有界. 对任意的 $g \in L^{q^{\prime}}$ 且 $g \geqslant 0$, 由 Fubini 定理可得

$$
\begin{aligned}
\int_{\partial \mathbb{D}} g(\zeta) A_{\mu}^{\sigma}(f)(\zeta)|d \zeta| & =\int_{\mathbb{D}} \int_{\partial \mathbb{D}} \chi_{\Gamma_{\sigma}(\zeta)}(z) g(\zeta)|f(z)||d \zeta| \frac{d \mu(z)}{1-|z|} \\
& =\int_{\mathbb{D}} \int_{I_{\sigma}(z)} g(\zeta)|d \zeta||f(z)| \frac{d \mu(z)}{1-|z|} \\
& \asymp \int_{\mathbb{D}} T_{\sigma}(g)(z)|f(z)| d \mu(z) \quad \text { (由 (2.5) 式). }
\end{aligned}
$$

令 $I$ 为圆周 $\partial \mathbb{D}$ 上的弧. 回顾对 $a \in \mathbb{D}$ 而言, 弧 $I_{\sigma}(a)$ 的定义:

$$
I_{\sigma}(a)=\left\{\zeta \in \partial \mathbb{D}: a \in \Gamma_{\sigma}(\zeta)\right\} .
$$

不难看出, 存在 $a \in \mathbb{D}$, 使得 $I_{\sigma}(a)=I$, 因而

$$
(1-|a|) \asymp\left|I_{\sigma}(a)\right|=|I| .
$$

定义以 $I$ 为底的 $\sigma$-帐管 (tent) 为

$$
\Lambda_{\sigma}(I)=\left\{z \in \mathbb{D}: \overline{I_{\sigma}(z)} \subset I\right\},
$$

这里 $\bar{E}$ 表示集合 $E$ 的闭包. 注意到在 $\lambda$-Carleson 测度的定义中, Carleson 方形 $S(I)$ 可换成 $\sigma$ - 帐篗 $\Lambda_{\sigma}(I)(\sigma>0$ 可以是任意固定的参数 $)$, 这个转换并不改变 $\lambda$-Carleson 测度的本质, 因为总存在着 Carleson 方形 $S\left(I_{1}\right)$ 和 $S\left(I_{2}\right)$, 使得

$$
S\left(I_{1}\right) \subset \Lambda_{\sigma}(I) \subset S\left(I_{2}\right), \quad\left|I_{1}\right| \asymp\left|I_{2}\right| \asymp|I| .
$$

记单位圆周 $\partial \mathbb{D}$ 上的弧 $I_{\sigma}(a)(=I)$ 的特征函数为 $g_{a}$, 易见

$$
\left\|g_{a}\right\|_{L^{q^{\prime}}}=c(1-|a|)^{1 / q^{\prime}} \asymp|I|^{1 / q^{\prime}},
$$

且当 $z \in \Lambda_{\sigma}(I)$ 时，有

$$
T_{\sigma}\left(g_{a}\right)(z)=1
$$

因此可得

$$
\int_{\Lambda_{\sigma}(I)}|f(z)| d \mu(z) \leqslant \int_{\mathbb{D}} T_{\sigma}\left(g_{a}\right)(z)|f(z)| d \mu(z) \leqslant C|I|^{1 / q^{\prime}}\|f\|_{p, \alpha} .
$$

在下面的论证中, 将引用 Khinchine 不等式, 这一技巧是由 Luecking 首先使 用的, 他用此方法 (在文献 [9] 中) 证明了本文引用的定理 B.

对 $t \in[0,1)$, 和 $j=1,2, \cdots$, 定义

$$
r_{j}(t)=r_{0}\left(2^{j} t\right)
$$

这里

$$
r_{0}(s)= \begin{cases}1, & 0 \leqslant s-[s]<1 / 2 \\ -1, & 1 / 2 \leqslant s-[s]<1 .\end{cases}
$$

Khinchine 不等式称: 对任意的固定参数 $0<p<\infty$, 存在 $c_{p}>0$, 使得

$$
c_{p}\left(\sum_{j=0}^{N}\left|c_{j}\right|^{2}\right)^{p / 2} \leqslant \int_{0}^{1}\left|\sum_{j=0}^{N} c_{j} r_{j}(t)\right|^{p} d t \leqslant \frac{1}{c_{p}}\left(\sum_{j=0}^{N}\left|c_{j}\right|^{2}\right)^{p / 2} .
$$


令 $\left\{z_{j}\right\}$ 为 $\mathbb{D}$ 内的 $\delta$-网格,

$$
m>\max \left\{-1,2\left(1-\frac{1}{p}\right)\right\}(>0), \quad f_{j}(z)=\frac{\left(1-\left|z_{j}\right|\right)^{m}}{\left(1-\overline{z_{j}} z\right)^{m+\frac{\alpha+2}{p}}} .
$$

由定理 $\mathrm{A}$ 可知, 当 $\delta>0$ 充分小时, 对任意给定的

$$
S=\left\{a_{j}\right\} \in l^{p},
$$

有

$$
f_{S}(z)=\sum_{j} a_{j} f_{j}(z) \in \mathcal{A}_{p}^{\alpha}
$$

且有

$$
\left\|f_{S}\right\|_{p, \alpha} \leqslant C\|S\|_{l^{p}} .
$$

令

$$
S(t)=\left\{a_{j} r_{j}(t)\right\}, \quad \forall t \in[0,1),
$$

显然有

$$
\|S(t)\|_{l^{p}}=\|S\|_{l^{p}}, \quad \forall t \in[0,1) .
$$

将估计 (3.3) 式中的 $f(z)$ 换成 $f_{S(t)}(z)$, 则有

$$
\int_{\Lambda_{\sigma}(I)}\left|f_{S(t)}(z)\right| d \mu(z) \leqslant C|I|^{1 / q^{\prime}}\left\|f_{S(t)}\right\|_{p, \alpha} \leqslant C|I|^{1 / q^{\prime}}\|S\|_{l^{p}} .
$$

将上面的不等式两边对 $t$ 在区间 $[0,1)$ 上积分, 然后用 Khinchine 不等式, 得到

$$
\int_{\Lambda_{\sigma}(I)}\left(\sum_{j}\left|a_{j}\right|^{2}\left|f_{j}(z)\right|^{2}\right)^{1 / 2} d \mu(z) \leqslant C|I|^{1 / q^{\prime}}\|S\|_{l^{p}} .
$$

由等价估计 (2.2) 式可得

$$
\mu\left(\tilde{D}_{j}\right)\left(1-\left|z_{j}\right|\right)^{-\frac{\alpha+2}{p}} \asymp \int_{\tilde{D}_{j}}\left|f_{j}(z)\right| d \mu(z) .
$$

因此, 用上面的估计可推导出

$$
\begin{aligned}
\sum_{j: \tilde{D}_{j} \subset \Lambda_{\sigma}(I)} \frac{\left|a_{j}\right| \mu\left(\tilde{D}_{j}\right)}{\left(1-\left|z_{j}\right|\right)^{\frac{\alpha+2}{p}}} & \asymp \sum_{j: \tilde{D}_{j} \subset \Lambda_{\sigma}(I)}\left|a_{j}\right| \int_{\tilde{D}_{j}}\left|f_{j}(z)\right| d \mu(z) \\
& \leqslant C \sum_{j: \tilde{D}_{j} \subset \Lambda_{\sigma}(I)} \int_{\tilde{D}_{j}}\left(\sum_{k}\left|a_{k}\right|^{2}\left|f_{k}(z)\right|^{2}\right)^{1 / 2} d \mu(z) \\
& \leqslant C L \int_{\Lambda_{\sigma}(I)}\left(\sum_{j}\left|a_{j}\right|^{2}\left|f_{j}(z)\right|^{2}\right)^{1 / 2} d \mu(z) \quad \text { (由 (2.3) 式) } \\
& \leqslant C|I|^{1 / q^{\prime}}\|S\|_{l^{p}} .
\end{aligned}
$$

再利用 $l^{p}$ 和 $l^{p^{\prime}}$ 的对偶关系, 可以进一步推出

$$
\left(\sum_{j: \tilde{D}_{j} \subset \Lambda_{\sigma}(I)}\left(\frac{\mu\left(\tilde{D}_{j}\right)}{\left(1-\left|z_{j}\right|\right)^{\frac{\alpha+2}{p}}}\right)^{p^{\prime}}\right)^{1 / p^{\prime}} \leqslant C|I|^{1 / q^{\prime}},
$$


这相当于

$$
\sum_{j: \tilde{D}_{j} \subset \Lambda_{\sigma}(I)}\left(\rho_{\alpha}(\mu)\left(z_{j}, 2 \delta\right)\right)^{p^{\prime}}\left(1-\left|z_{j}\right|\right)^{\alpha+2} \leqslant C|I|^{p^{\prime} / q^{\prime}} .
$$

另一方面, 令

$$
\tau=\sigma_{4 \delta}=(\sigma+2) e^{8 \delta}-2
$$

我们断言

$$
\tilde{D}_{j} \cap \Lambda_{\tau}(I) \neq \emptyset \Longrightarrow \tilde{D}_{j} \subset \Lambda_{\sigma}(I)
$$

这等价于: 如果存在 $w \in \tilde{D}_{j} \cap \Lambda_{\tau}(I)$, 则对任意的 $z \in \tilde{D}_{j}$, 都有 $\overline{I_{\sigma}(z)} \subset I$. 事实 上, 这样的 $w$ 显然属于 $D(z, 4 \delta)$, 进而可由引理 2.3 导出 $I_{\sigma}(z) \subset I_{\tau}(w)$. 再因为 $w \in \Lambda_{\tau}(I)$, 所以又有 $\overline{I_{\tau}(w)} \subset I$, 因此 $\overline{I_{\sigma}(z)} \subset I$ 成立.

由上述断言可得

$$
\begin{aligned}
& \int_{\Lambda_{\tau}(I)}\left(\rho_{\alpha}(\mu)(z, \delta)\right)^{\frac{p}{p-1}} d A_{\alpha}(z) \\
\leqslant & \sum_{j: D_{j} \cap \Lambda_{\tau}(I) \neq \emptyset} \int_{D_{j}}\left(\rho_{\alpha}(\mu)(z, \delta)\right)^{\frac{p}{p-1}} d A_{\alpha}(z) \\
\leqslant & C \sum_{j: D_{j} \cap \Lambda_{\tau}(I) \neq \emptyset}\left(\rho_{\alpha}(\mu)\left(z_{j}, 2 \delta\right)\right)^{p^{\prime}}\left(1-\left|z_{j}\right|\right)^{\alpha+2} \quad \text { (由 (2.6) 及 }(2.1) \text { 式) } \\
\leqslant & C \sum_{j: \tilde{D}_{j} \subset \Lambda_{\sigma}(I)}\left(\rho_{\alpha}(\mu)\left(z_{j}, 2 \delta\right)\right)^{p^{\prime}}\left(1-\left|z_{j}\right|\right)^{\alpha+2} \\
\leqslant & C|I|^{p^{\prime} / q^{\prime}} \text { (由 (3.4) 式). }
\end{aligned}
$$

很显然

$$
\frac{p^{\prime}}{q^{\prime}}=\frac{p(q-1)}{q(p-1)}
$$

从而 $(2 b) \Rightarrow(2 c)$ 得证.

为了证明 $(2 \mathrm{c}) \Rightarrow(2 \mathrm{~d})$, 注意到只要 $0<t \leqslant \delta$, 测度 $\left(\rho_{\alpha}(\mu)(z, t)\right)^{\frac{p}{p-1}} d A_{\alpha}(z)$ 就 是一个 $\frac{p(q-1)}{q(p-1)}$-Carleson 测度, 这是因为, 由估计 (2.6) 式可得

$$
\rho_{\alpha}(\mu)(z, t) \leqslant C \rho_{\alpha}(\mu)(z, \delta),
$$

故此假设 $t>\delta$.

令 $s>t-\delta$, 由引理 2.8 及 Hölder 不等式, 可得

$$
\left(\rho_{\alpha}(\mu)(z, t)\right)^{\frac{p}{p-1}} \leqslant \frac{C}{|D(z, s)|_{\alpha}} \int_{D(z, s)}\left(\rho_{\alpha}(\mu)(w, \delta)\right)^{\frac{p}{p-1}} d A_{\alpha}(w),
$$

因此, 可将 $(2 \mathrm{c}) \Rightarrow(2 \mathrm{~d})$ 的证明归结为证明某一类 “平均算子” 具有保持 Carleson 测度不变的性质. 因为这一结果有其自身的意义, 稍后将单独陈述及证明它.

最后证明 $(2 \mathrm{~d}) \Rightarrow(2 \mathrm{a})$. 令

$$
t>0, \quad \sigma=3 e^{2 t}-2,
$$


由引理 2.7 可得

$$
A_{\mu}(f)(\zeta) \leqslant C \int_{\Gamma_{\sigma}(\zeta)}|f(z)| \frac{\rho_{\alpha}(\mu)(z, t) d A_{\alpha}(z)}{1-|z|} .
$$

令 $g \in L^{q^{\prime}}$ 且 $g \geqslant 0$, 由 Fubini 定理及定理 2.4, 可得

$$
\begin{aligned}
& \int_{\partial \mathbb{D}} g(\zeta) A_{\mu}(f)(\zeta)|d \zeta| \\
\leqslant & C \int_{\partial \mathbb{D}} g(\zeta) \int_{\Gamma_{\sigma}(\zeta)}|f(z)| \frac{\rho_{\alpha}(\mu)(z, t) d A_{\alpha}(z)}{1-|z|}|d \zeta| \\
\asymp & \int_{\mathbb{D}} T_{\sigma}(g)(z)|f(z)| \rho_{\alpha}(\mu)(z, t) d A_{\alpha}(z) \\
\leqslant & C \int_{\mathbb{D}} P(g)(z)|f(z)| \rho_{\alpha}(\mu)(z, t) d A_{\alpha}(z) \\
\leqslant & C\|f\|_{p, \alpha}\left(\int_{\mathbb{D}}|P(g)(z)|^{p^{\prime}}\left(\rho_{\alpha}(\mu)(z, t)\right)^{p^{\prime}} d A_{\alpha}(z)\right)^{1 / p^{\prime}} \\
\leqslant & C\|f\|_{p, \alpha}\left(\int_{\mathbb{D}}|\mathcal{H}(g)(z)|^{p^{\prime}}\left(\rho_{\alpha}(\mu)(z, t)\right)^{p^{\prime}} d A_{\alpha}(z)\right)^{1 / p^{\prime}} \\
\leqslant & C\|f\|_{p, \alpha}\|\mathcal{H}(g)\|_{L^{q^{\prime}}} \quad(\text { 由定理 D }) \\
\leqslant & C\|f\|_{p, \alpha}\|g\|_{L^{q^{\prime}}},
\end{aligned}
$$

因此

$$
\left\|A_{\mu}(f)\right\|_{L^{q}} \leqslant C\|f\|_{p, \alpha} .
$$

引理 3.1 令 $\alpha>-1, \lambda, t>0, \mu$ 为单位圆盘 $\mathbb{D}$ 上的非负测度, 则 $\mu$ 是 $\lambda$-Carleson 测度 (或紧的 $\lambda$-Carleson 测度) 的充要条件为测度 $\rho_{\alpha}(\mu)(z, t) d A_{\alpha}(z)$ 是 $\lambda$-Carleson 测度 (或紧的 $\lambda$-Carleson 测度).

证 首先证明充分性. 令 $I$ 是单位圆周 $\partial \mathbb{D}$ 上的弧, 再令 $\sigma>0,\left\{z_{j}\right\}$ 是单 位圆盘 $\mathbb{D}$ 内的 $\delta$-网格且 $\delta \leqslant t / 2$, 又令

$$
\tau=(\sigma+2) e^{4 \delta}-2 .
$$

类似于定理 2 证明中的断言, 可以得到

$$
D_{j} \cap \Lambda_{\tau}(I) \neq \emptyset \Longrightarrow D_{j} \subset \Lambda_{\sigma}(I) .
$$

将所有这些假设及断言综合到一起, 就有

$$
\begin{aligned}
\mu\left(\Lambda_{\tau}(I)\right) & \leqslant \sum_{j: D_{j} \cap \Lambda_{\tau}(I) \neq \emptyset} \mu\left(D_{j}\right) \\
& \asymp \sum_{j: D_{j} \cap \Lambda_{\tau}(I) \neq \emptyset} \rho_{\alpha}(\mu)\left(z_{j}, \delta\right)\left|D_{j}\right|_{\alpha} \\
& \leqslant C \sum_{j: D_{j} \subset \Lambda_{\sigma}(I)} \int_{D_{j}} \rho_{\alpha}(\mu)(z, 2 \delta) d A_{\alpha}(z) \\
& \leqslant C L \int_{\Lambda_{\sigma}(I)} \rho_{\alpha}(\mu)(z, t) d A_{\alpha}(z) .
\end{aligned}
$$


“必要性”可以用类似的办法证明. 事实上, 如果

$$
\eta=(\sigma+2) e^{4 \delta+2 t}-2,
$$

则由引理 2.3 可得

$$
D_{j} \cap \Lambda_{\eta}(I) \neq \emptyset \Longrightarrow D\left(z_{j}, \delta+t\right) \subset \Lambda_{\sigma}(I) .
$$

进而由引理 2.8 可得

$$
\begin{aligned}
\int_{D_{j}} \rho_{\alpha}(\mu)(z, t) d A_{\alpha}(z) & \leqslant C \rho_{\alpha}(\mu)\left(z_{j}, \delta+t\right)\left|D_{j}\right|_{\alpha} \\
& \asymp \mu\left(D\left(z_{j}, \delta+t\right)\right),
\end{aligned}
$$

因此

$$
\begin{aligned}
\int_{\Lambda_{\eta}(I)} \rho_{\alpha}(\mu)(z, t) d A_{\alpha}(z) & \leqslant \sum_{j: D_{j} \cap \Lambda_{\eta}(I) \neq \emptyset} \int_{D_{j}} \rho_{\alpha}(\mu)(z, t) d A_{\alpha}(z) \\
& \leqslant \sum_{j: D\left(z_{j}, \delta+t\right) \subset \Lambda_{\sigma}(I)} \mu\left(D\left(z_{j}, \delta+t\right)\right) \\
& \leqslant C L \mu\left(\Lambda_{\sigma}(I)\right) \quad \text { (由 (2.3) 式). }
\end{aligned}
$$

定理 3 令 $\alpha>-1,1 \leqslant q<p \leqslant \infty$. 对于单位圆盘 $\mathbb{D}$ 上的非负测度 $\mu$ 而 言, 下列各陈述等价:

(3a) $A_{\mu}$ 从 $\mathcal{A}_{p}^{\alpha}$ 到 $L^{q}$ 有界.

(3b) 存在 $\sigma>0$, 使得 $A_{\mu}^{\sigma}$ 从 $\mathcal{A}_{p}^{\alpha}$ 到 $L^{q}$ 有界.

(3c) 存在 $\delta, \sigma>0$, 使得 $\alpha$ - 密度函数 $\rho_{\alpha}(\mu)(z, \delta)$ 满足条件

$$
\int_{\Gamma_{\sigma}(\zeta)}\left(\rho_{\alpha}(\mu)(z, \delta)\right)^{\frac{p}{p-1}} \frac{d A_{\alpha}(z)}{1-|z|} \in L^{\frac{(p-1) q}{p-q}} .
$$

(3d) 对任意固定的 $t, \sigma>0, \alpha$ - 密度函数 $\rho_{\alpha}(\mu)(z, t)$ 具有上述性质.

注 3 我们可以得到关于 $A_{\mu}^{\sigma}$ 是从 $\mathcal{A}_{p}^{\alpha}$ 到 $L^{q}$ 上的紧算子的相关结果, 只需 将定理 3 的各陈述中的 “有界” 换为 “紧”, 且将 (3c) 中 $\alpha$ - 密度函数 $\rho_{\alpha}(\mu)(z, \delta)$ 满 足的条件换为

$$
\left\|\int_{\Gamma_{\sigma}(\zeta) \backslash D_{\rho}}\left(\rho_{\alpha}(\mu)(z, \delta)\right)^{\frac{p}{p-1}} \frac{d A_{\alpha}(z)}{1-|z|}\right\|_{L}^{\frac{(p-1) q}{p-q}} \rightarrow 0 \quad(\rho \rightarrow 1),
$$

这里

$$
0<\rho<1, \quad D_{\rho}=\{z:|z|<\rho\}
$$

其证明和下面定理 3 的证明类似.

定理 3 的证 我们将按照下列顺序进行证明:

(1) $(3 \mathrm{c}) \Rightarrow(3 \mathrm{~d})$;

(2) $1 \leqslant q<p=\infty$ 时, (3b) $\Rightarrow$ (3c), (3d) $\Rightarrow$ (3a);

(3) $1 \leqslant q<p<\infty$ 时, (3b) $\Rightarrow$ (3c), (3d) $\Rightarrow$ (3a).

其余要证的都很容易. 
(1) 假设 (3c) 成立. 为方便起见, 记

$$
w_{t}(z)=\left(\rho_{\alpha}(\mu)(z, t)\right)^{\frac{p}{p-1}}, \quad \lambda=\frac{(p-1) q}{p-q} .
$$

根据假设, 存在 $\delta, \sigma>0$, 使得

$$
\int_{\Gamma_{\sigma}(\zeta)} w_{\delta}(z) \frac{d A_{\alpha}(z)}{1-|z|} \in L^{\lambda}
$$

只需证明对任意固定的 $t, \tau>0$, 函数 $w_{t}$ 满足

$$
\int_{\Gamma_{\tau}(\zeta)} w_{t}(z) \frac{d A_{\alpha}(z)}{1-|z|} \in L^{\lambda}
$$

令 $g \in L^{\lambda^{\prime}}$ (因为 $\lambda \geqslant 1$, 所以 $\lambda^{\prime}$ 存在), 且 $g \geqslant 0$. 我们知道

$$
\left\|N_{\tau} P(g)\right\|_{L^{\lambda^{\prime}}} \leqslant C\|g\|_{L^{\lambda^{\prime}}},
$$

因而由对偶原理, 只需证明

$$
\int_{\partial \mathbb{D}} g(\zeta) \int_{\Gamma_{\tau}(\zeta)} w_{t}(z) \frac{d A_{\alpha}(z)}{1-|z|}|d \zeta| \leqslant C \int_{\partial \mathbb{D}} N_{\tau} P(g)(\zeta) \int_{\Gamma_{\sigma}(\zeta)} w_{\delta}(z) \frac{d A_{\alpha}(z)}{1-|z|}|d \zeta| .
$$

由 Fubini 定理, 上述不等式等价于

$$
\int_{\mathbb{D}} T_{\tau}(g)(z) w_{t}(z) d A_{\alpha}(z) \leqslant C \int_{\mathbb{D}} T_{\tau} N_{\tau} P(g)(z) w_{\delta}(z) d A_{\alpha}(z) .
$$

当 $t \leqslant \delta$ 时, 上述估计显然成立. 令 $t>\delta$, 再令 $s>t-\delta$, 由估计 (3.5) 式可得

$$
w_{t}(z) \leqslant \frac{C}{|D(z, s)|_{\alpha}} \int_{D(z, s)} w_{\delta}(u) d A_{\alpha}(u) .
$$

将调和函数的平均值性质用于非负调和函数 $P(g)$, 再用估计 (2.1) 和 (2.2) 式, 得

$$
P(g)(u) \asymp \frac{1}{|D(u, s)|_{\alpha}} \int_{D(u, s)} P(g)(z) d A_{\alpha}(z),
$$

因此

$$
\begin{aligned}
\int_{\mathbb{D}} T_{\tau}(g)(z) w_{t}(z) d A_{\alpha}(z) & \leqslant C \int_{\mathbb{D}} P(g)(z) \frac{C}{|D(z, s)|_{\alpha}} \int_{D(z, s)} w_{\delta}(u) d A_{\alpha}(u) d A_{\alpha}(z) \\
& \asymp \int_{\mathbb{D}} P(g)(u) w_{\delta}(u) d A_{\alpha}(u) \\
& \leqslant \int_{\mathbb{D}} T_{\tau} N_{\tau} P(g)(z) w_{\delta}(z) d A_{\alpha}(z) \quad \text { (由定理 2.5). }
\end{aligned}
$$

$(3 \mathrm{c}) \Rightarrow(3 \mathrm{~d})$ 得证.

(2) 设 $1 \leqslant q<p=\infty$, 要证明 (3b) $\Rightarrow$ (3c) 与 (3d) $\Rightarrow$ (3a).

假设 (3b) 成立, 即算子 $A_{\mu}^{\sigma}$ 从 $\mathcal{A}_{\infty}^{\alpha}$ 到 $L^{q}$ 有界. 首先可得 $A_{\mu}^{\sigma}(1) \in L^{q}$.

选一个 $\delta>0$, 使得

$$
2\left(e^{6 \delta}-1\right)<\sigma
$$

则

$$
\tau=(\sigma+2) e^{-6 \delta}-2>0
$$


对于任意的单位圆盘 $\mathbb{D}$ 内的 $\delta$-网格 $\left\{z_{j}\right\}$, 由引理 2.3 (类似的推论已在定理 1 的 证明中给出) 可得

$$
D_{j} \cap \Gamma_{\tau}(\zeta) \neq \emptyset \Longrightarrow \tilde{D}_{j} \subset \Gamma_{\sigma}(\zeta)
$$

从而有

$$
\begin{aligned}
& \int_{\Gamma_{\tau}(\zeta)} \rho_{\alpha}(\mu)(z, \delta) \frac{d A_{\alpha}(z)}{1-|z|} \\
\leqslant & \sum_{j: D_{j} \cap \Gamma_{\tau}(\zeta) \neq \emptyset} \int_{D_{j}} \rho_{\alpha}(\mu)(z, \delta) \frac{d A_{\alpha}(z)}{1-|z|} \\
\leqslant & C \sum_{j: D_{j} \cap \Gamma_{\tau}(\zeta) \neq \emptyset} \rho_{\alpha}(\mu)\left(z_{j}, 2 \delta\right) \frac{\left|D_{j}\right|_{\alpha}}{1-\left|z_{j}\right|} \quad \text { 由 (2.6) 及 }(2.2) \text { 式) } \\
\leqslant & C \sum_{j: \tilde{D}_{j} \subset \Gamma_{\sigma}(\zeta)} \frac{\mu\left(\tilde{D}_{j}\right)}{1-\left|z_{j}\right|} \quad \text { (由 }(2.1) \text { 式) } \\
\leqslant & C L \int_{\Gamma_{\sigma}(\zeta)} \frac{d \mu(z)}{1-|z|} \quad \text { (由 (2.2) 及 }(2.3) \text { 式) } \\
= & C A_{\mu}^{\sigma}(1)(\zeta) .
\end{aligned}
$$

据此可以推断

$$
\int_{\Gamma_{\tau}(\zeta)} \rho_{\alpha}(\mu)(z, \delta) \frac{d A_{\alpha}(z)}{1-|z|} \in L^{q}
$$

$(3 b) \Rightarrow(3 c)$ 得证.

对于 $1 \leqslant q<p=\infty$ 的情形, (3d) $\Rightarrow(3 \mathrm{a})$ 是引理 2.7 的推论. 事实上, 令

$$
\sigma=3 e^{2 t}-2 \text {, }
$$

则有

$$
\begin{aligned}
A_{\mu}(f)(\zeta) & \leqslant C \int_{\Gamma_{\sigma}(\zeta)}|f(z)| \frac{\rho_{\alpha}(\mu)(z, t) d A_{a}(z)}{1-|z|} \\
& \leqslant C\|f\|_{\mathcal{A}_{\infty}^{\alpha}} \int_{\Gamma_{\sigma}(\zeta)} \frac{\rho_{\alpha}(\mu)(z, t) d A_{a}(z)}{1-|z|} .
\end{aligned}
$$

(3) 考虑 $1 \leqslant q<p<\infty$ 的情形. 由于 $q=1$ 即定理 2.1 , 故可假设 $q>1$.

假设 $A_{\mu}^{\sigma}$ 从 $\mathcal{A}_{p}^{\alpha}$ 到 $L^{q}$ 有界. 令 $g \in L^{q^{\prime}}$ 且 $g \geqslant 0$. 类似于定理 2 的证明, 得

$$
\begin{aligned}
\int_{\mathbb{D}} T_{\sigma}(g)(z)|f(z)| d \mu(z) & \asymp \int_{\partial \mathbb{D}} g(\zeta) A_{\mu}^{\sigma}(f)(\zeta)|d \zeta| \\
& \leqslant C\|g\|_{L^{q^{\prime}}}\|f\|_{p, \alpha} .
\end{aligned}
$$

令 $\left\{z_{j}\right\}$ 是单位圆盘 $\mathbb{D}$ 内的 $\delta$-网格且 $\delta>0$ 充分小, 再令 $m>2$ 及

$$
f_{j}(z)=\frac{\left(1-\left|z_{j}\right|\right)^{m}}{\left(1-\overline{z_{j}} z\right)^{m+\frac{\alpha+2}{p}}} .
$$

类似于定理 2 的证明，应用上述不等式及 Khinchine 不等式，可以得到

$$
\int_{\mathbb{D}} T_{\sigma}(g)(z)\left(\sum_{j}\left|a_{j}\right|^{2}\left|f_{j}(z)\right|^{2}\right)^{1 / 2} d \mu(z) \leqslant C\left\|\left\{a_{j}\right\}\right\|_{l^{p}}\|g\|_{L^{q^{\prime}}} .
$$


对 $z \in \tilde{D}_{j}\left(=D\left(z_{j}, 2 \delta\right)\right)$, 由等价估计 (2.2) 式可得

$$
\left(\sum_{k}\left|a_{k}\right|^{2}\left|f_{k}(z)\right|^{2}\right)^{1 / 2} \geqslant\left|a_{j}\right|\left|f_{j}(z)\right| \asymp \frac{\left|a_{j}\right|}{\left(1-\left|z_{j}\right|\right)^{\frac{\alpha+2}{p}}} .
$$

由于 $\mathbb{D}$ 被 $\left\{\tilde{D}_{j}\right\}$ 所覆盖, 且此覆盖使得估计 (2.3) 式对于一致的常数 $L>0$ 成立, 因而有

$$
\begin{aligned}
& \sum_{j} \frac{\left|a_{j}\right|}{\left(1-\left|z_{j}\right|\right)^{\frac{\alpha+2}{p}}} \int_{\tilde{D}_{j}} T_{\sigma}(g)(z) d \mu(z) \\
\leqslant & C \sum_{j} \int_{\tilde{D}_{j}} T_{\sigma}(g)(z)\left(\sum_{k}\left|a_{k}\right|^{2}\left|f_{k}(z)\right|^{2}\right)^{1 / 2} d \mu(z) \\
\leqslant & C L \int_{\mathbb{D}} T_{\sigma}(g)(z)\left(\sum_{j}\left|a_{j}\right|^{2}\left|f_{j}(z)\right|^{2}\right)^{1 / 2} d \mu(z) \\
\leqslant & C\left\|\left\{a_{j}\right\}\right\|_{l^{p}}\|g\|_{L^{q^{\prime}}} .
\end{aligned}
$$

再由 $l^{p}$ 和 $l^{p^{\prime}}$ 的对偶关系, 就可得到

$$
\sum_{j}\left(\frac{1}{\left(1-\left|z_{j}\right|\right)^{\frac{\alpha+2}{p}}} \int_{\tilde{D}_{j}} T_{\sigma}(g)(z) d \mu(z)\right)^{p^{\prime}} \leqslant C\|g\|_{L^{q^{\prime}}}^{p^{\prime}} .
$$

假设

$$
h \in L^{q^{\prime}}, \quad h \geqslant 0, \quad \tau=\sigma_{2 \delta}=(\sigma+2) e^{4 \delta}-2,
$$

由定理 2.5 可得, 如果 $w \in \tilde{D}_{j}$, 则

$$
|P(h)(w)| \leqslant T_{\sigma} N_{\tau} P(h)\left(z_{j}\right)
$$

因此

$$
\int_{\tilde{D}_{j}}|P(h)(w)|^{p^{\prime}} d A_{\alpha}(w) \leqslant\left|\tilde{D}_{j}\right|_{\alpha}\left(T_{\sigma} N_{\tau} P(h)\left(z_{j}\right)\right)^{p^{\prime}} .
$$

令

$$
g=N_{\tau} P N_{\tau} P(h),
$$

由于 $q^{\prime}>1$, 算子 $N_{\tau} P$ 在 $L^{q^{\prime}}$ 上有界, 因此

$$
\|g\|_{L^{q^{\prime}}} \leqslant C\|h\|_{L^{q^{\prime}}} .
$$

再由定理 2.4 和 2.5 , 得到当 $w \in D(z, 2 \delta)$, 或等价地, 当 $z \in D(w, 2 \delta)$ 时, 有

$$
\begin{aligned}
T_{\sigma} N_{\tau} P(h)(w) & \leqslant C\left|P N_{\tau} P(h)(w)\right| \\
& \leqslant C T_{\sigma} N_{\tau} P N_{\tau} P(h)(z)=C T_{\sigma}(g)(z) .
\end{aligned}
$$

将上面的不等式两边对 $d \mu(z)$ 在 $D(w, 2 \delta)$ 上积分, 得到

$$
T_{\sigma} N_{\tau} P(h)(w) \mu(D(w, 2 \delta)) \leqslant C \int_{D(w, 2 \delta)} T_{\sigma}(g)(z) d \mu(z) .
$$


综合上述各不等式，得

$$
\begin{aligned}
& \left(\rho_{\alpha}(\mu)\left(z_{j}, 2 \delta\right)\right)^{p^{\prime}} \int_{\tilde{D}_{j}}|P(h)(w)|^{p^{\prime}} d A_{\alpha}(w) \\
\leqslant & \left(\mu\left(\tilde{D}_{j}\right)\left|\tilde{D}_{j}\right|_{\alpha}^{-1}\right)^{p^{\prime}}\left|\tilde{D}_{j}\right|_{\alpha}\left(T_{\sigma} N_{\tau} P(h)\left(z_{j}\right)\right)^{p^{\prime}} \\
\leqslant & C\left|\tilde{D}_{j}\right|_{\alpha}^{1-p^{\prime}}\left(\int_{\tilde{D}_{j}} T_{\sigma}(g)(z) d \mu(z)\right)^{p^{\prime}} \\
\asymp & \left(\frac{1}{\left(1-\left|z_{j}\right|\right)^{\frac{\alpha+2}{p}}} \int_{\tilde{D}_{j}} T_{\sigma}(g)(z) d \mu(z)\right)^{p^{\prime}} \text { (由 (2.1) 式). }
\end{aligned}
$$

再引用估计 (3.6) 式, 就有

$$
\sum_{j}\left(\rho_{\alpha}(\mu)\left(z_{j}, 2 \delta\right)\right)^{p^{\prime}} \int_{\tilde{D}_{j}}|P(h)(w)|^{p^{\prime}} d A_{\alpha}(w) \leqslant C\|g\|_{L^{q^{\prime}}}^{p^{\prime}} \leqslant C\|h\|_{L^{q^{\prime}}}^{p^{\prime}} .
$$

进一步由估计 (2.3) 及 (2.8) 式, 可推出

$$
\begin{aligned}
& \int_{\mathbb{D}}|P(h)(w)|^{p^{\prime}}\left(\rho_{\alpha}(\mu)(w, \delta)\right)^{p^{\prime}} d A_{\alpha}(w) \\
\leqslant & \sum_{j} \int_{D_{j}}|P(h)(w)|^{p^{\prime}}\left(\rho_{\alpha}(\mu)(w, \delta)\right)^{p^{\prime}} d A_{\alpha}(w) \\
\leqslant & C \sum_{j}\left(\rho_{\alpha}(\mu)\left(z_{j}, 2 \delta\right)\right)^{p^{\prime}} \int_{D_{j}}|P(h)(w)|^{p^{\prime}} d A_{\alpha}(w) \\
\leqslant & C\|h\|_{L^{q^{\prime}}}^{p^{\prime}},
\end{aligned}
$$

因此, 由定理 2.2 可得到 (3c).

$(3 d) \Rightarrow(3 a)$ 是引理 2.7 及 Hölder 不等式的推论. 事实上, 令

$$
t>0, \quad \sigma=3 e^{2 t}-2>1,
$$

由引理 2.7 和 Hölder 不等式 $(p>1)$ 可得

$$
\begin{aligned}
A_{\mu}(f)(\zeta) & \leqslant C \int_{\Gamma_{\sigma}(\zeta)}|f(z)| \rho_{\alpha}(\mu)(z, t) \frac{d A_{\alpha}(z)}{1-|z|} \\
& \leqslant C\left(\int_{\Gamma_{\sigma}(\zeta)}|f(z)|^{p} \frac{d A_{\alpha}(z)}{1-|z|}\right)^{1 / p}\left(\int_{\Gamma_{\sigma}(\zeta)}\left[\rho_{\alpha}(\mu)(z, t)\right]^{p^{\prime}} \frac{d A_{\alpha}(z)}{1-|z|}\right)^{1 / p^{\prime}} .
\end{aligned}
$$

再次运用 Hölder 不等式得

$$
\begin{aligned}
\int_{\partial \mathbb{D}}\left|A_{\mu}(f)(\zeta)\right|^{q}|d \zeta| \leqslant & C\left(\int_{\partial \mathbb{D}} \int_{\Gamma_{\sigma}(\zeta)}|f(z)|^{p} \frac{d A_{\alpha}(z)}{1-|z|}|d \zeta|\right)^{q / p} \\
& \times\left(\int_{\partial \mathbb{D}}\left(\left[\rho_{\alpha}(\mu)(z, t)\right]^{p^{\prime}} \frac{d A_{\alpha}(z)}{1-|z|}\right)^{\left(q / p^{\prime}\right)(p / q)^{\prime}}|d \zeta|\right)^{1 /(p / q)^{\prime}} \\
& \asymp\|f\|_{p, \alpha}^{q}\left\|\int_{\Gamma_{\sigma}(\zeta)}\left[\rho_{\alpha}(\mu)(z, t)\right]^{p^{\prime}} \frac{d A_{\alpha}(z)}{1-|z|}\right\|_{L^{\frac{(p-1) q}{p-q}}}^{(p-1) q / p}
\end{aligned}
$$

其中的等价符号 “こ” 是由

$$
\frac{1}{(p / q)^{\prime}}=\frac{p-q}{p}, \quad\left(q / p^{\prime}\right)(p / q)^{\prime}=\frac{(p-1) q}{p-q}
$$


得到的. $(3 \mathrm{~d}) \Rightarrow(3 \mathrm{a})$ 证毕.

定理 4 令 $\alpha>-1, q<p \leqslant \infty, 0<q<1$. 对单位圆盘 $\mathbb{D}$ 上的非负测度 $\mu$ 而言, 下列各陈述等价:

(4a) 对任意固定的 $\sigma>0, A_{\mu}^{\sigma}$ 从 $\mathcal{A}_{p}^{\alpha}$ 到 $L^{q}$ 有界.

(4b) 对任意固定的 $\tau>0$, 单位圆盘 $\mathbb{D}$ 内的 $\delta$-网格 $\left\{z_{j}\right\}$ 及 $l^{p}$ 中的任意元素 $\left\{a_{j}\right\}, \alpha$ - 密度函数 $\rho_{\alpha}(\mu)(z, \delta)$ 满足

$$
\int_{\partial \mathbb{D}}\left(\sum_{j: z_{j} \in \Gamma_{\tau}(\zeta)}\left|a_{j}\right| \frac{\rho_{\alpha}(\mu)\left(z_{j}, \delta\right)}{\left(1-\left|z_{j}\right|\right)^{(\alpha+2)\left(\frac{1}{p}-1\right)+1}}\right)^{q}|d \zeta| \leqslant C\left\|\left\{a_{j}\right\}\right\|_{l^{p}}^{q} .
$$

注 4 当 $p=\infty$ 时, (4b) 等价于 $\alpha$ - 密度函数 $\rho_{\alpha}(\mu)(z, \delta)$ 满足

$$
\int_{\Gamma_{\sigma}(\zeta)}\left(\rho_{\alpha}(\mu)(z, \delta)\right) \frac{d A_{\alpha}(z)}{1-|z|} \in L^{q} .
$$

我们可以得到关于 $A_{\mu}^{\sigma}$ 是从 $\mathcal{A}_{p}^{\alpha}$ 到 $L^{q}$ 上的紧算子的相关结果, 只需将定理 4 的 各陈述中的 “有界” 换为 “紧”, 且将 (4b) 中 $\alpha$ - 密度函数 $\rho_{\alpha}(\mu)(z, \delta)$ 应满足的条件 换为

$$
\sup _{\left\|\left\{a_{j}\right\}\right\|_{l^{p}}=1}\left\|\sum_{j: z_{j} \in \Gamma_{\tau}(\zeta) \backslash D_{\rho}}\left|a_{j}\right| \frac{\rho_{\alpha}(\mu)\left(z_{j}, \delta\right)}{\left(1-\left|z_{j}\right|\right)^{(\alpha+2)\left(\frac{1}{p}-1\right)+1}}\right\|_{L^{q}} \rightarrow 0 \quad(\rho \rightarrow 1),
$$

其证明和下面定理 4 的证明类似.

为了证明定理 4 , 需要引用 Kalton ${ }^{[14]}$ 的一个结果. 对次 Banach 空间 $\mathcal{X}$ 而言, $K$ - 范数 $\|\cdot\|$ 是一个从 $\mathcal{X}$ 到 $[0, \infty)$ 的函数, 它具有通常范数所应满足的除了三角 不等式之外的所有性质. 虽然三角不等式对 $K$ - 范数不成立, 但是对于所有的 $x, y \in \mathcal{X}, \mathcal{K}$ - 范数 $\|\cdot\|$ 满足

$$
\|x+y\| \leqslant K(\|x\|+\|y\|) .
$$

定理 $\mathbf{E}$ 令 $0<r<s<\infty, K>0$, 则存在正常数 $c$ 和 $C$, 使得对任意次 Banach 空间 $\mathcal{X}$ 及其上的 $K$ - 范数 $\|\cdot\|$, 和任意正整数 $m$ 及任意 $x_{1}, x_{2}, \cdots, x_{m} \in \mathcal{X}$, 下面的不等式成立:

$$
\begin{aligned}
c\left(\int_{0}^{1}\left\|\sum_{j} r_{j}(t) x_{j}\right\|^{r} d t\right)^{1 / r} & \leqslant\left(\int_{0}^{1}\left\|\sum_{j} r_{j}(t) x_{j}\right\|^{s} d t\right)^{1 / s} \\
& \leqslant C\left(\int_{0}^{1}\left\|\sum_{j} r_{j}(t) x_{j}\right\|^{r} d t\right)^{1 / r} .
\end{aligned}
$$

定理 4 的证 假设对任意固定的 $\sigma>0, A_{\mu}^{\sigma}$ 从 $\mathcal{A}_{p}^{\alpha}$ 到 $L^{q}$ 有界. 令

$$
D_{\rho}=\{z:|z|<\rho\}, \quad 0<\rho<1 .
$$

对单位圆盘 $\mathbb{D}$ 上的解析函数 $f$, 定义

$$
\|f\|_{\zeta, \sigma, \rho}=\int_{\Gamma_{\sigma}(\zeta) \cap D_{\rho}}|f(z)| \frac{d \mu(z)}{1-|z|} .
$$

显然, 对 $K=1,\|\cdot\|_{\zeta, \sigma, \rho}$ 满足定理 $\mathrm{E}$ 中的假设, 因此对 $0<q<1$ 和 $\left\{f_{j}\right\} \subset \mathcal{A}_{p}^{\alpha}$, 
由定理 $\mathrm{E}$ 可得

$$
\left(\int_{0}^{1}\left\|\sum_{j} a_{j} r_{j}(t) f_{j}(z)\right\|_{\zeta, \sigma, \rho}^{q} d t\right)^{1 / q} \asymp \int_{0}^{1}\left\|\sum_{j} a_{j} r_{j}(t) f_{j}(z)\right\|_{\zeta, \sigma, \rho} d t .
$$

由于 Khinchine 不等式保证了

$$
\int_{0}^{1}\left|\sum_{j} a_{j} r_{j}(t) f_{j}(z)\right| d t \asymp\left(\sum_{j}\left|a_{j}\right|^{2}\left|f_{j}(z)\right|^{2}\right)^{1 / 2},
$$

因此得到

$$
\int_{\Gamma_{\sigma}(\zeta) \cap D_{\rho}}\left(\sum_{j}\left|a_{j}\right|^{2}\left|f_{j}(z)\right|^{2}\right)^{1 / 2} \frac{d \mu(z)}{1-|z|} \asymp\left(\int_{0}^{1}\left\|\sum_{j} a_{j} r_{j}(t) f_{j}(z)\right\|_{\zeta, \sigma, \rho}^{q} d t\right)^{1 / q} .
$$

令 $\left\{a_{j}\right\}$ 为 $l^{p}$ 中的元素, 且令

$$
f_{j}(z)=\frac{\left(1-\left|z_{j}\right|\right)^{m}}{\left(1-\overline{z_{j}} z\right)^{m+\frac{\alpha+2}{p}}}, \quad m>2, \quad j=1,2, \cdots .
$$

根据定理 $A$, 得

$$
f_{t}(z)=\sum_{j} a_{j} r_{j}(t) f_{j}(z) \in \mathcal{A}_{p}^{\alpha}
$$

且对所有的 $t \in[0,1)$, 有

$$
\left\|f_{t}\right\|_{p, \alpha} \leqslant C\left\|\left\{a_{j}\right\}\right\|_{l^{p}} .
$$

将上式两边的 $q$ 次方对 $|d \zeta|$ 积分, 得

$$
\int_{\partial \mathbb{D}}\left(\int_{\Gamma_{\sigma}(\zeta) \cap D_{\rho}}\left(\sum_{j}\left|a_{j}\right|^{2}\left|f_{j}(z)\right|^{2}\right)^{1 / 2} \frac{d \mu(z)}{1-|z|}\right)^{q}|d \zeta| \leqslant C\left\|\left\{a_{j}\right\}\right\|_{l^{p}}^{q} .
$$

特别注意, 上式中的常数 $C$ 与 $\rho$ 无关. 令 $\rho \rightarrow 1$, 并用定理 2 证明中的类似方法 可得

$$
\int_{\partial \mathbb{D}}\left(\sum_{j: D_{j} \subset \Gamma_{\sigma}(\zeta)}\left|a_{j}\right| \frac{\mu\left(D_{j}\right)}{\left(1-\left|z_{j}\right|\right)^{\frac{\alpha+2}{p}+1}}\right)^{q}|d \zeta| \leqslant C\left\|\left\{a_{j}\right\}\right\|_{l^{p}}^{q} .
$$

对任意固定的 $\tau, \delta>0$, 令

$$
\sigma=(\tau+2) e^{2 \delta}-2,
$$

则由引理 2.3 有

$$
z_{j} \in \Gamma_{\tau}(\zeta) \Longrightarrow D_{j} \subset \Gamma_{\sigma}(\zeta)
$$

因此, 用上面的估计就可推出 (4b).

现假设 (4b) 成立. 对任意固定的 $\sigma>0$, 令

$$
\tau=(\sigma+2) e^{2 \delta}-2,
$$

我们知道

$$
D_{j} \cap \Gamma_{\sigma}(\zeta) \neq \emptyset \Longrightarrow z_{j} \in \Gamma_{\tau}(\zeta)
$$


对单位圆盘 $\mathbb{D}$ 上任意的解析函数 $f$, 有

$$
\begin{aligned}
A_{\mu}^{\sigma}(f)(\zeta) & \leqslant C \sum_{j: D_{j} \cap \Gamma_{\sigma}(\zeta) \neq \emptyset} \sup _{z \in D_{j}}|f(z)| \frac{\mu\left(D_{j}\right)}{1-\left|z_{j}\right|} \\
& \asymp \sum_{j: D_{j} \cap \Gamma_{\sigma}(\zeta) \neq \emptyset}\left(1-\left|z_{j}\right|\right)^{\frac{\alpha+2}{p}} \sup _{z \in D_{j}}|f(z)| \frac{\rho_{\alpha}(\mu)\left(z_{j}, \delta\right)}{\left(1-\left|z_{j}\right|\right)^{(\alpha+2)\left(\frac{1}{p}-1\right)+1}} \\
& \leqslant \sum_{j: z_{j} \in \Gamma_{\tau}(\zeta)}\left(1-\left|z_{j}\right|\right)^{\frac{\alpha+2}{p}} \sup _{z \in D_{j}}|f(z)| \frac{\rho_{\alpha}(\mu)\left(z_{j}, \delta\right)}{\left(1-\left|z_{j}\right|\right)^{(\alpha+2)\left(\frac{1}{p}-1\right)+1} .}
\end{aligned}
$$

只需证明序列

$$
\left\{\left(1-\left|z_{j}\right|\right)^{\frac{\alpha+2}{p}} \sup _{z \in D_{j}}|f(z)|\right\} \in l^{p}
$$

且它的 $l^{p}$ 范数能被 $C\|f\|_{p, \alpha}$ 所控制. 如果 $p=\infty$, 这是显然的. 若 $p<\infty$, 由估 计 (3.2) 式得

$$
\sup _{z \in D_{j}}|f(z)|^{p} \leqslant \frac{C}{\left|\tilde{D}_{j}\right|_{\alpha}} \int_{\tilde{D}_{j}}|f(w)|^{p} d A_{\alpha}(w) .
$$

因此

$$
\begin{aligned}
& \sum_{j}\left(1-\left|z_{j}\right|\right)^{\alpha+2} \sup _{z \in D_{j}}|f(z)|^{p} \\
\leqslant & C \sum_{j} \int_{\tilde{D}_{j}}|f(w)|^{p} d A_{\alpha}(w) \\
\leqslant & C L \int_{\mathbb{D}}|f(w)|^{p} d A_{\alpha}(w) .
\end{aligned}
$$

最后我们指出, 用类似本文的方法, 可定义和研究更广一类的区域积分算 子

$$
\left(\int_{\Gamma_{\sigma}(\zeta)}|f(z)|^{r} \frac{d \mu(z)}{1-|z|}\right)^{1 / r}
$$

\section{参考文献}

1 Cohn W S. Generalized area operators on Hardy spaces. J Math Anal Appl, 1997, 216(1): 112-121

2 Duren P L. Extension of a theorem of Carleson. Bull Amer Math Soc, 1969, 75: 143-146

3 Carleson L. An interpolation problem for bounded analytic functions. Amer J Math, 1958, 80: 921-930

4 Garnett J B. Bounded Analytic Functions. New York/London: Academic Press, 1982

5 Luecking D. Trace ideal criteria for Toeplitz operators. J Funct Anal, 1987, 73(2): 345-368

6 Wu Z. Carleson measures and multipliers for Dirichlet spaces. J Funct Anal, 1999, 169(1): 148-163

7 Coifman R R, Rochberg R. Representation theorems for holomorphic and harmonic functions in $L^{p}$. Astérisque, 1980, 77: 11-66

8 Rochberg R. Decomposition theorems for Bergman spaces and their applications. In: Power S C, ed. Operators and Function Theory (Lancaster, 1984). NATO Adv Sci Inst Ser C Math Phys Sci, 153. Dordrecht: Reidel, 1985. 225-277 
9 Luecking D. Embedding theorems for spaces of analytic functions via Khinchine's inequality. Michigan Math J, 1993, 40(2): 333-358

10 Wu Z, Xie C. $Q$ spaces and Morrey spaces. J Funct Anal, 2003, 201(1): 282-297

11 Xiao J. Holomorphic $Q$ Classes. Lecture Notes in Mathematics, Vol 1767. Berlin: Springer-Verlag, 2001

12 Videnskii I V. On an analogue of Carleson measures. Dokl Akad Nauk SSSR, 1988, 298: 1042-1047; Soviet Math Dokl, 1988, 37: 186-190

13 Luecking D. Embedding derivatives of Hardy spaces into Lebesgue spaces. Proc London Math Soc, 1991, 63(3): 595-619

14 Kalton N. Convexity, type and the three space problem. Studia Math, 1980/1981, 69(3): 247-287 